\title{
Evolution and decay of gravity wavefields in weak-rotating environments: a laboratory study
}

\author{
Pedro Rojas ${ }^{1} \cdot$ Hugo N. Ulloa ${ }^{2}$ (D) Yarko Niño ${ }^{3}$
}

Received: 25 September 2017 / Accepted: 16 June 2018

(c) Springer Nature B.V. 2018

\begin{abstract}
Gravity waves are prominent physical features that play a fundamental role in transport processes of stratified aquatic ecosystems. In a two-layer stratified basin, the equations of motion for the first vertical mode are equivalent to the linearised shallow water equations for a homogeneous fluid. We adopted this framework to examine the spatiotemporal structure of gravity wavefields weakly affected by the background rotation of a single-layer system of equivalent thickness $h_{\ell}$, via laboratory experiments performed in a cylindrical basin mounted on a turntable. The wavefield was generated by the release of a diametral linear tilt of the air-water interface, $\eta_{\ell}$, inducing a basin-scale perturbation that evolved in response to the horizontal pressure gradient and the rotation-induced acceleration. The basin-scale wave response was controlled by an initial perturbation parameter, $\mathcal{A}_{*}=\eta_{0} / h_{\ell}$, where $\eta_{0}$ was the initial displacement of the air-water interface, and by the strength of the background rotation controlled by the Burger number, $S$. We set the experiments to explore a transitional regime from moderate- to weak-rotational environments, $0.65 \leq S \leq 2$, for a wide range of initial perturbations, $0.05 \leq \mathcal{A}_{*} \leq 1.0$. The evolution of $\eta_{\ell}$ was registered over a diametral plane by recording a laser-induced optical fluorescence sheet and using a capacitive sensor located near the lateral boundary. The evolution of the gravity wavefields showed substantial variability as a function of the rotational regimes and the radial position. The results demonstrate that the strength of rotation and nonlinearities control the bulk decay rate of the basin-scale gravity waves. The ratio between the experimentally estimated damping timescale, $T_{d}$, and the seiche period of the basin, $T_{g}$, has a median value of $T_{d} / T_{g} \approx 11$, a maximum value of $T_{d} / T_{g} \approx 10^{3}$ and a minimum value of $T_{d} / T_{g} \approx 5$. The results of this study are significant for the understanding the dynamics of gravity waves in waterbodies weakly affected by Coriolis acceleration, such as mid- to small-size lakes.
\end{abstract}

Keywords Basin-scale gravity waves $\cdot$ Rotational regimes $\cdot$ Damping rate of gravity waves

Hugo N. Ulloa

hugo.ulloa@epfl.ch

Extended author information available on the last page of the article 


\section{Introduction}

Wind-driven basin-scale gravity waves mediate a fundamental transport mechanism [36, 59] and can modulate biogeochemical processes that control the environmental quality in lake systems [39-41, 55]. Depending on the size and latitude of the lake, the spatiotemporal structure of gravity waves may or may not be affected by the local Coriolis acceleration caused by the Earth's rotation. The effect of Coriolis on the basin-scale gravity wave dynamics can be quantified by the Burger number, $S \equiv R_{\ell} / R[2]$, defined as the ratio of the Rossby radius of deformation, $R_{\ell}=c_{\ell} / f$, to the relevant horizontal length-scale of the waterbody, $R$ (for instance, half the width of the basin or half the longest fetch length) $[2,27,37]$, where $c_{\ell}$ is the longwave celerity and $f$ is the inertial frequency. Hence, as $S \rightarrow 0$, the background rotation becomes strongly dominant in the flow dynamics. Conventionally, it has been established that when $S>1$ the Coriolis effect becomes weak and can play a negligible role in the momentum balance $[25,27$, $38,42,56]$.

In the case of large lakes, (e.g., the Laurentian Great Lakes in North America, and Lakes Geneva and Constance in West Europe), Coriolis acceleration strongly affects large-scale oscillations allowing the existence of two major classes of large-scale gravity waves, widely known as Kelvin and Poincaré waves (rotating gravity waves) [14]. A Kelvin wave is a cyclonic sub/super-inertial oscillation trapped along the lateral boundary, whose amplitude, $\eta_{\ell}(t, x)$, decays exponentially from the coast to the interior of the lake and whose horizontal flow has a strong alongshore component. A Poincaré wave, by contrast, is an anti-cyclonic super-inertial oscillation, defined by a cell-like structure whose maximum amplitude is located in the centre of the cell, that propagates mainly across the interior of the basin. As the basin's size and/or the latitude decreases, the effect of rotation becomes weaker and rotating gravity waves converge to either surface or internal seiches [25, 30, 37]. However, besides basin size and latitude, the background stratification of the waterbody also plays a role in the rotation effect. Seasonal variations in the density structure cause changes in the effect of rotation on large-scale internal waves as the celerity of the waves changes [3], which in turn has a direct impact on the seasonal variability of physical and biochemical processes in lakes [3, 39].

The dynamics of Kelvin and Poincaré waves has been widely studied in systems where rotation plays a first-order role, $S \sim \mathcal{O}\left(10^{-2}-10^{-1}\right)$, via field observations in large- to mid-size lakes [2, 10, 11, 44, 46]. Additionally, numerical simulations, both in real and ideal basins [4, 13, 17, 47, 54], and laboratory experiments [33, 45, 53, 57] have provided a robust understanding of the basin-scale wave dynamics in systems with rotational regimes characterised by $0.1 \leq S<1$. In particular, laboratory-scale results show that rotational regimes with $S \leq 0.5$ are associated with strong degeneration and decay of the basin-scale internal gravity waves via the boundary friction and nonlinear processes associated with the gravest sub-inertial Kelvin wave [45, 48, 53, 57]. In contrast, the evolution and decay of basin-scale gravity waves in the regime where the gravest Kelvin wave is no longer sub-inertial, $S>1 / \sqrt{2}$ [2, 49], have not been explored with particular interest. A few works have reported laboratory experiments in this regime, including the works by Wake et al. [57, 58] and Ulloa et al. [53] who performed experiments with Burger numbers $S=0.75,0.8,1.0$. In addition to the limited number of experiments in the super-inertial regime, the above mentioned experimental works $[53,57,58]$ focused on rotational regimes characterised by $S \leq 0.5$, in which the gravity wavefield is composed of both sub-inertial and super-inertial gravity waves. 
Characterising gravity wavefields in systems weakly affected by rotation will provide a better understanding of physical processes and timescales that govern mid- to small-size lakes.

The objective of this experimental study is to examine the evolution and bulk decay rate of gravity wavefields in the transitional regime, in which the fundamental Kelvin wave becomes super-inertial and the Rossby radius of deformation, $R_{\ell}$, becomes comparable or larger than the waterbody horizontal length-scale, $R$. For this purpose, we set the Burger number in the range $0.65 \leq S \leq 2$, allowing us to explore the transition from wavefields dominated by sub- and super-inertial waves to wavefields entirely characterised by superinertial waves. This particular rotational regime is dynamically relevant, since previous studies have shown that the degeneration processes of basin-scale waves are sensitive and dependent on the background rotation and the nature of the wave (sub-inertial or superinertial waves) $[16,47,53]$. We use a cylindrical basin mounted on a turntable (Fig. 1a-c) to generate and register the spatiotemporal structure of gravity wavefields. The experimental conditions are based on the theoretical results of Csanady [15] and Stocker and Imberger [49]. The above authors obtained analytical solutions for the evolution of a linear and inviscid gravity wavefield and a geostrophic flow resulting from the release of a linearly tilted interface in a single- and a two-layer rotating circular basin of constant depth on an $f$-plane. For practical reasons, we adopt a single-layer system to perform the laboratory experiments. Notice that the evolution equations for the first vertical mode in a densitystratified system are equivalent to the equations of a homogeneous fluid [19, 28, 43]. To match the rotational regimes between a two- and a single-layer system, one needs to choose appropriate equivalent water depths and angular rotations.

The remainder of the paper is outlined as follows. In Sect. 2, we formulate the controlling parameters of the basin-scale gravity wavefield. In Sect. 3, we introduce the experimental method and the experimental set. In Sect. 4, we examine the gravity wavefield near the lateral boundary and interior zones, and we present the decay rate of the basin-scale waves regarding the controlling parameters. Finally, in Sect 5, we discuss the scope of this study in the context of gravity waves in lakes, and we summarise the main results.

\section{Formulation}

In this section, we introduce the physical parameters that control the basin-scale gravity wave dynamics in the rotating system and the theoretical initial value problem of the laboratory experiments.

\subsection{Single-layer dynamics}

In linear theory, the equations of motion for the first vertical mode of a density-stratified fluid (in particular a two-layer system) in a flat and shallow basin are equivalent to the linearised equations for a single-layer fluid [19, 28, 43]. For this case, the horizontal spatial structure of the internal normal modes is analogous to the barotropic or surface normal modes. Thus, a single-layer system (in the inviscid regime) is equivalent to a two-layer system with a rigid-lid approximation, assuming that internal modes are negligibly distorted by barotropic modes. Based on this assumption, the longwave celerity is $c_{\ell}=\sqrt{g h_{\ell}}$, where $h_{\ell}$ is the equivalent depth (the case of air-water density interface). To match the desired rotational regime or Burger number, we control the angular velocity of the system, 
$\Omega_{z}$. This framework is adopted to design a single-layer experiment, which is substantially simpler than a two-layer stratified laboratory experiment.

The linearised equations of motion and the dispersion relations for gravity waves in a flat and shallow circular basin on an $f$-plane have been extensively investigated; for a detailed derivation of these equations the readers are referred to Csanady [14] (for the twolayer case), Shimizu and Imberger [48] (for the general density-stratified case), and Johnson and Grimshaw [28] (in the context of geostrophic adjustment in a homogeneous fluid). The spatial structure and the frequency of the Kelvin and the Poincare waves are obtained by solving the eigenvalue problem based on the linearised equations and lateral boundary conditions [14]. Here, we denote the Kelvin and Poincaré waves in terms of their radial and azimuthal wavenumbers, $n_{r}$ and $n_{\theta}$, as $K\left(n_{r}, n_{\theta}\right)$ and $P\left(n_{r}, n_{\theta}\right)$, and whose dimensional frequencies are labelled $\omega_{K\left(n_{r}, n_{\theta}\right)}$ and $\omega_{P\left(n_{r}, n_{\theta}\right)}$, respectively. Similarly, we denote the purely radial waves as $B\left(n_{r}, n_{\theta}=0\right)$, hereafter Bessel modes. In Sect. 2.3, we further introduce the geostrophic component of the ideal initial condition for the laboratory experiments.

\subsection{Dimensionless numbers}

Three dimensionless parameters determine the dynamics regime of the shallow, basin-scale gravity wavefield:

$$
\mathcal{A}_{*} \equiv \frac{\eta_{0}}{h_{\ell}}, \quad S \equiv \frac{R_{\ell}}{R}=\frac{c_{\ell}}{f R}, \quad \mathcal{T}_{v f} \equiv \frac{T_{v}}{T_{f}}=\frac{\eta_{0}^{2} v^{-1}}{T_{f}}
$$

The first parameter, $\mathcal{A}_{*}$, defined by the ratio of maximum initial displacement of the density interface, $\eta_{0}$, to the equivalent layer thickness, $h_{\ell}$, controls the initial perturbation of the basin-scale gravity waves. Indeed, $\mathcal{A}_{*}^{2}$ is a measure of the initial potential energy available for exciting the gravity wavefield. As $\mathcal{A}_{*} \rightarrow 1$ (maximum value in this study), the resulting basin-scale waves are more energetic and prone to nonlinearities. In contrast, the wavefield becomes linear as $\mathcal{A}_{*} \rightarrow 0$. Hereafter, $\mathcal{A}_{*}$ is denoted as the perturbation parameter.

The second parameter, $S$, corresponds to the Burger number (already defined in Sect. 1), the ratio of the Rossby radius of deformation, $R_{\ell}=c_{\ell} / f$, and radius of the cylindrical basin, $R$, where $f=2 \Omega_{z}$ is the inertial frequency or Coriolis parameter, and $\Omega_{z}$ is the basin angular velocity. Rotation becomes more important in the wave dynamics as $S \rightarrow 0$; on the contrary, rotation effect becomes weaker as $S$ increases. Note that $S^{-1}$ is analogous to the Kelvin number defined as $K \equiv R / R_{\ell}$ [28].

The third parameter, $\mathcal{T}_{v f}$, is the ratio between viscous diffusion and rotation effect, where $v$ denotes the kinematic viscosity. $\mathcal{T}_{v f}$ is interpreted as the ratio of the timescale required by vertical diffusion to acts over $\eta_{0}$ to the inertial period, $T_{f}=2 \pi / f$. This parameter can be expressed in terms of the Ekman number, $E k \equiv \nu T_{f} / h_{\ell}^{2}$, and the perturbation parameter, $\mathcal{A}_{*}, \mathcal{T}_{v f} \equiv \mathcal{A}_{*}^{2} E k^{-1}$. High values of $\mathcal{T}_{v f}$ are associated with low viscous-controlled waves affected by rotation. On the contrary, vertical diffusion and viscous damping processes become significant as $\mathcal{T}_{v f} \rightarrow 0$. In this work, we denote $\mathcal{T}_{v f}$ as the inverse Ekman parameter (also called rotation number).

A fourth not-independent parameter is adopted to compare nonlinear steepening and gravity timescales, $\mathcal{T}_{s g} \equiv T_{s} / T_{g}$. Here, the steepening timescale is defined as $T_{s}=4 R / \Delta c_{\ell}$, where $\Delta c_{\ell} \equiv \max \left\{c_{\ell}\left(\eta_{0}\right)\right\}-\min \left\{c_{\ell}\left(\eta_{0}\right)\right\}$ is the maximum difference of long wave celerity in the basin controlled by the initial interface perturbation, $\mathcal{A}_{*}$. We denote $\mathcal{T}_{s g}$ as the steepening parameter and is determined by $\mathcal{A}_{*}$ as $\mathcal{T}_{s g}=1 /\left(\sqrt{1+\mathcal{A}_{*}}-\sqrt{1-\mathcal{A}_{*}}\right)$. This 
parameter is usually $\mathcal{T}_{s g} \gg 1$ and tends to $\mathcal{T}_{s g} \rightarrow 1 / \sqrt{2}$ when $\mathcal{A}_{*} \rightarrow 1$. Therefore, a stronger wave steepening process is expected as $\mathcal{T}_{s g} \rightarrow 1 / \sqrt{2}$.

\subsection{Initial value problem}

The initial condition consists of a linear interface tilt in a direction $\theta_{0}$ with slope $C$ and null wavefield:

$$
\begin{gathered}
\eta_{\ell}\left(t_{0}, r, \theta\right) / \eta_{0} \equiv C r \cos \left(\theta-\theta_{0}\right) / h_{\ell}, \\
\mathbf{v} \equiv 0,
\end{gathered}
$$

where $t_{0}$ is the initial time, $C=h_{\ell} /\left(R \mathcal{A}_{*}\right), \mathbf{v}$ is the horizontal transport, which satisfies $\mathbf{v} \cdot \hat{r}=0$ on the lateral boundary, and $r$ and $\theta$ are polar coordinates. Note that by choosing $\theta_{0}=0$ in (2) the interface displacement $\eta_{\ell}$ is tilted in the $x$-direction, $r \cos (\theta)$. The general linear and inviscid rotating long wave solution for (2) is [49]:

$$
\begin{gathered}
\eta_{\ell}(t, r, \theta) / \eta_{0}=Q_{c} \mathcal{A}_{*}^{-1} \cos \left(\theta-\theta_{0}\right)-\mathcal{A}_{*}^{-1} \sum_{n_{r}=1}^{\infty} a_{n_{r}} D_{n_{r}} A_{n_{r}}, \\
Q_{c}=\left(r / R_{\ell}\right) S, \\
D_{n_{r}}=-\sigma_{n_{r}} \sin \left(\theta-\theta_{0}-\sigma_{n_{r}}\left(t-t_{0}\right) f\right), \\
a_{n_{r}}=\left(\sigma_{n_{r}}-1\right) /\left(1+\sigma_{n_{r}}-S^{-2} \sigma_{n_{r}}^{3}\right), \\
A_{n_{r}}=I_{1}\left(r \alpha_{\ell, n_{r}}\right) / I_{1}\left(R \alpha_{\ell, n_{r}}\right),
\end{gathered}
$$

where $\sigma_{n_{r}} \equiv \omega_{n_{r}} / f$ is the dimensionless frequency of the radial mode $n_{r}$ and azimuthal mode $n_{\theta}=1, \alpha_{n_{r}} \equiv\left(\sigma_{n_{r}}^{2}-1\right)^{1 / 2} / R_{\ell}$ denotes the radial wavenumber and $I_{1}$ is the modified Bessel function of the first kind [1] that defines the radial modal structure of the first azimuthal mode $n_{\theta}=1$. The vertical displacement, $\eta_{\ell}$, in Eq. (3a) is characterised by the linear superposition of a time-independent term that represents a geostrophic component and an infinite sum of normal modes including the cyclonic Kelvin waves and anticyclonic Poincaré waves. The first term results from the adjustment between the rotating fluid and the initial pressure gradient that relaxes to a steady state geostrophic balance, also denoted as a geostrophic mode, $G\left(n_{r}, n_{\theta}\right)$, which has a frequency of zero [19, 28, 43]. The initial displacement defined in Eq. (2a) forces only the fundamental azimuthal mode 1 and all the possible radial modes $[8,15,49]$. In our experiments, the angle $\theta_{0}$ and the initial time $t_{0}$ were set to zero.

Stocker and Imberger [49] investigated the partitioning of energy among the fundamental Kelvin wave, Poincare wave, and the geostrophic component as a function of the Burger number. As the Burger number increases, the geostrophic component becomes substantially weaker relative to the Kelvin and Poincaré waves. In the rotational regime of our study, $0.65 \leq S \leq 2.0$; although the Kelvin wave stores more energy than the Poincaré wave, this energy decreases as a function of $S$ while the energy of the Poincaré wave increases. Figure 1d-p show an example of the spatial structure of the initially energised/ 
non-energised normal modes (gravity waves) and the geostrophic component in the cylindrical domain (Fig. 2).

Although the solution (3a) cannot be reproduced via laboratory experiments, this defines the linear and inviscid evolution of the initially energised gravity waves. Due to nonlinearities and diffusion (degeneration mechanisms), one expects differences between the evolution of the air-water interface in the laboratory and the analytical solution (3a).

\section{Method and experimental set}

The experiments were carried out on a rotating table whose rotation speed varies from 0 to 6 r.p.m. A cylindrical transparent acrylic basin, with a diameter of $1.8 \mathrm{~m}$ and depth of 0.5 $\mathrm{m}$, was mounted on the turntable. The turntable has a synchronised hydraulic arm, which allows tilting the cylindrical basin and releasing it to the horizontal position, in a cushioned form and in a short time $\left(t_{r} \sim 1 \mathrm{~s}\right)$. In the interior of the cylindrical basin a layer of water thickness $h_{\ell}=5 \times 10^{-2} \mathrm{~m}$ and density $\rho_{\ell}=999 \mathrm{~kg} \mathrm{~m}^{-3}$ was placed (Fig. 3). The facility is located at the F. J. Domínguez Hydraulics Laboratory, University of Chile.

Each experiment was performed in two stages. First, the dimensionless parameters of the experiment $S$ and $\mathcal{A}_{*}$ were fixed. $S$ was set by varying the angular velocity of the rotating table to obtain the desired inertial frequency, $f$. $\mathcal{A}_{*}$ was obtained by measuring the linear inclination of the basin from the hydraulic arm with respect to the horizontal base (Fig. 3). Second, once the initial condition of the system was reached (see Sect. 2.2), the basin was released quickly but damped to the horizontal position. This new configuration of the fluid volume induces an adjustment of the water surface in response to the horizontal barotropic gradient and the background rotation, ideally exciting all the radial modes and only the fundamental azimuthal mode of the gravitational waves of the system. The vertical evolution of the free surface was recorded by the combination of an optical method of laser-induced fluorescence and a capacitive sensor to measure the water level; both methods mounted on the rotational system. For the second method, we used a capacitive sensor (Mark ChurchillControls, model Wave Monitor) that recorded the water level at $100 \mathrm{~Hz}$ in a single point, at the external boundary of the basin, where $\eta_{0}(t=0, r \approx 0.98 R, \theta=-\pi / 2)=0$, as shown in Fig. 3. This sensor was calibrated to measure water height in the range of amplitudes expected in the experiments, $0 \leq \eta_{\ell} \leq 0.05 \mathrm{~m}$. For the first method, a fluorescent sheet (along the diameter of the basin) was created using fluorescein type B to dye the water layer and an array of 5 green wavelength lasers, arranged as shown in Fig. 3. The diametrical fluorescent plane was recorded at $25 \mathrm{~Hz}$ by a CCD camera of resolution $5184 \times 3456$ pixels for an area $1.8 \times 0.5 \mathrm{~m}^{2}$. The optical method was calibrated by relating the gauge measure on the lateral boundary with the position of the air-water interface in the pixelcoordinate system associated to the CCD. It is important to notice that the optical method allowed recording the gravity wavefield in the interior, while the capacitive sensor allowed registering the gravity wavefield at the near-lateral boundary.

The experimental set considered nine values for the Burger number, $S \in[0.65 ; 0.70 ; 0.75 ; 0.80 ; 0.85 ; 0.90 ; 0.95 ; 1.0 ; 2.0]$, and six values for the initial perturbation, $\mathcal{A}_{*} \in[0.05 ; 0.2 ; 0.4 ; 0.6 ; 0.8 ; 1.0]$, generating a total of 54 experiments. In the case of the steepening parameter, the range was $\mathcal{T}_{s g} \in[1 / \sqrt{2} ; 20.0]$, while the range of the inverse Ekman parameter was $\mathcal{T}_{v f} \in[0.320 ; 394]$. The magnitude of measurement errors was determined for each variable recorded according to the instrumental precision, the calibrations performed, and by propagation of uncertainty. The presented results include 
the quantification of their errors derived from the propagation of errors at their source. The strongest rotational regime explored in this study is denoted as moderate-rotational regime (from now on M-RR), $S=0.65$, while the weakest rotational regime is denoted as weakrotational regime (from now on W-RR), $S=2.0$.

\section{Results}

In Sects. 4.1 and 4.2, we present a subset of six experiments to illustrate the gravity wavefield structure as a function of the rotational regime, $\mathcal{S}$, and the initial perturbation, $\mathcal{A}_{*}$. Table 1 summarises the dimensionless parameters of these experiments. The first three experiments are characterised as M-RR, $S=0.65$, with three different initial perturbations, $\mathcal{A}_{*}=0.2,0.4,1.0$, respectively. The second three experiments are characterised as W-RR, $S=2.0$, with the same three initial perturbations, $\mathcal{A}_{*}=0.2,0.4,1.0$, respectively. Here, we analyse the wavefields via power spectral densities (PSD) [6] and wavelet spectra (WS) [52], computed from time series of the interface displacement, $\eta_{\ell}(t) / h_{\ell}$, in three locations: at the near-lateral boundary, $r / R \approx 0.98$; at $r / R \approx 0.5$; and the centre of the basin, $r / R \approx 0$. We separate the results of the gravity wavefields in two zones. Time series of $\eta_{\ell} / h_{\ell}$ are presented in terms of the basin-scale wave period $T_{g}=4 R / c_{\ell}$, while the frequency in the spectra (PSD and WS) is normalised by the respective inertial frequency, $f$.

We first examine the near-lateral boundary gravity wavefields $(r / R \approx 0.98)$ in Sect. 4.1, then look at the interior gravity wavefields $(r / R \approx 0.5$ and $r / R \approx 0)$ in Sect. 4.2. The linear wavefield is classified by comparing experimental frequencies obtained from spectral energy peaks with the theoretical linear frequencies derived from the solution of the eigenvalue problem in a cylindrical domain [14]. Table 2 summarises the theoretical frequencies examined in this work. At the end of this section, we turn our focus to estimating the decay rate of basin-scale waves for the whole experimental set (54) in terms of the dimensionless parameters introduced in Sect. 2.2.

\subsection{Near-lateral boundary gravity wavefield}

Figure 4 shows the time series and PSD of $\eta_{\ell}$ at $r / R \approx 0.98$ for the above-described subset of experiments. Each time series includes a close-up of the first five seiche periods, $T_{g}$ (see Fig. 4a-f). PSDs of the M-RR and W-RR are shown in Fig. 4g, h, respectively.

In the M-RR, $S=0.65$, time series of $\eta_{\ell}(t) / h_{\ell}$ show that large initial perturbations, $\mathcal{A}_{*}$, are associated with strong nonlinear steepening and wave dispersion of the basinscale wave (compare close-up Fig. $4 \mathrm{a}, \mathrm{c}$, e). For $\mathcal{A}_{*}=0.2$, dispersive processes on the

Table 1 Parameters of subset of experiments examined in Sects. 4.1 and 4.2

\begin{tabular}{llllr}
\hline Experiment & $\mathcal{A}_{*}$ & \multicolumn{1}{c}{$S$} & $\mathcal{T}_{s g}$ & \multicolumn{1}{c}{$\mathcal{T}_{v f}$} \\
\hline Exp 1.1 & 0.2 & 0.65 & 4.97 & 15.75 \\
Exp 1.2 & 0.4 & 0.65 & 2.45 & 63.00 \\
Exp 1.3 & 1.0 & 0.65 & 0.71 & 393.78 \\
Exp 2.1 & 0.2 & 2.00 & 4.97 & 5.15 \\
Exp 2.2 & 0.4 & 2.00 & 2.45 & 20.48 \\
Exp 2.3 & 1.0 & 2.00 & 0.71 & 128.98 \\
\hline
\end{tabular}


Table 2 Theoretical dimensionless frequencies, $\sigma_{(\cdot)} \equiv \omega_{(\cdot)} / f$

\begin{tabular}{llr}
\hline Dimensionless frequency & M-RR & W-RR \\
& $S=0.65$ & $S=2.0$ \\
\hline$\sigma_{K(1,1)}$ & 0.904 & 3.309 \\
$\sigma_{P(1,1)}$ & 1.748 & 4.154 \\
$\sigma_{K(1,2)}$ & 3.573 & 10.673 \\
$\sigma_{P(1,2)}$ & 3.640 & 10.746 \\
$\sigma_{K(1,3)}$ & 5.625 & 17.088 \\
$\sigma_{P(1,3)}$ & 5.651 & 17.116 \\
$\sigma_{K(2,1)}$ & 1.696 & 5.763 \\
$\sigma_{P(2,1)}$ & 2.453 & 6.514 \\
$\sigma_{K(2,2)}$ & 4.425 & 13.400 \\
$\sigma_{P(2,2)}$ & 4.519 & 13.498 \\
$\sigma_{K(2,3)}$ & 19.943 \\
$\sigma_{P(2,3)}$ & 6.536 & 19.985 \\
$\sigma_{K P}^{-}$ & 6.577 & 0.838 \\
$\sigma_{K P}^{+}$ & 0.844 & 7.453 \\
$\sigma_{B(1,0)}$ & 2.651 & 7.728 \\
$\sigma_{B(2,0)}$ & 2.684 & 14.067 \\
$\sigma_{B(3,0)}$ & 4.669 & 20.372 \\
\hline
\end{tabular}

basin-scale wave are observed in the third wave period (Fig. 4a), while for $\mathcal{A}_{*}=0.4$, wave dispersion is found in the second wave period (Fig. 4c). In the case of $\mathcal{A}_{*}=1.0$, a strong dispersion is observed during the second period, but the first period already shows nonlinear dispersive effects (Fig. 4e). The timescales of the observed steepening and dispersion processes are consistent with the expected dynamics in most of the experiments. In the case of Exp. 1.1 (Fig. 4a), $\mathcal{T}_{s g}=4.97<\mathcal{T}_{v f}=15.75$, which predicts that the wave steepening starts during the fourth seiche period before viscous effects control the wavefield dynamics. However, wave steepening and dispersion were observed during the third seiche period. In the case of Exp. 1.2 (Fig. $4 \mathrm{c}$ ), $\mathcal{T}_{s g}=2.45<\mathcal{T}_{v f}=63.0$, predicting a steepening process during the second seiche period, consistent with the observation of the steepening and wave dispersion. Similarly, in Exp. 1.3 (Fig. 4e), $\mathcal{T}_{s g}=0.71<\mathcal{T}_{v f}=393.78$, the steepening parameter shows agreement with the start of the steepening process.

In the M-RR, basin-scale Kelvin waves are still characterised by a dominant azimuthal propagation with a pseudo-1D structure (along azimuthal axis), similar to the wave propagation observed in non-rotating channels $[9,25]$. However, we observe that after a certain time, the wavefield loses the regular solitary-type train wave pattern found in the non-rotating laboratory studies (see for instance time series figures in [9, 25]). Indeed, time series in Fig. $4 a, c$, e show a significant change in the interface-displacement pattern after roughly five seiche periods.

We expect that as the basin-scale wave radiates energy to higher azimuthal modes (see Fig. 1 initially unexcited modes with $n_{\theta}>1$ ), cell-type circulations will enrich the complexity of the wavefield in the near-lateral boundary zone. PSD in Fig. $4 \mathrm{~g}$ shows that the most energetic peak is close to the theoretical sub-inertial Kelvin wave ( $\boldsymbol{\nabla}$ marker) along with a broad spectrum of energy peaks associated with super-inertial, sub-azimuthal modes of Kelvin and Poincaré waves (triangles and circle markers, respectively). We observe that the larger the initial perturbation, $\mathcal{A}_{*}$, the higher the energy content in the high-frequency 

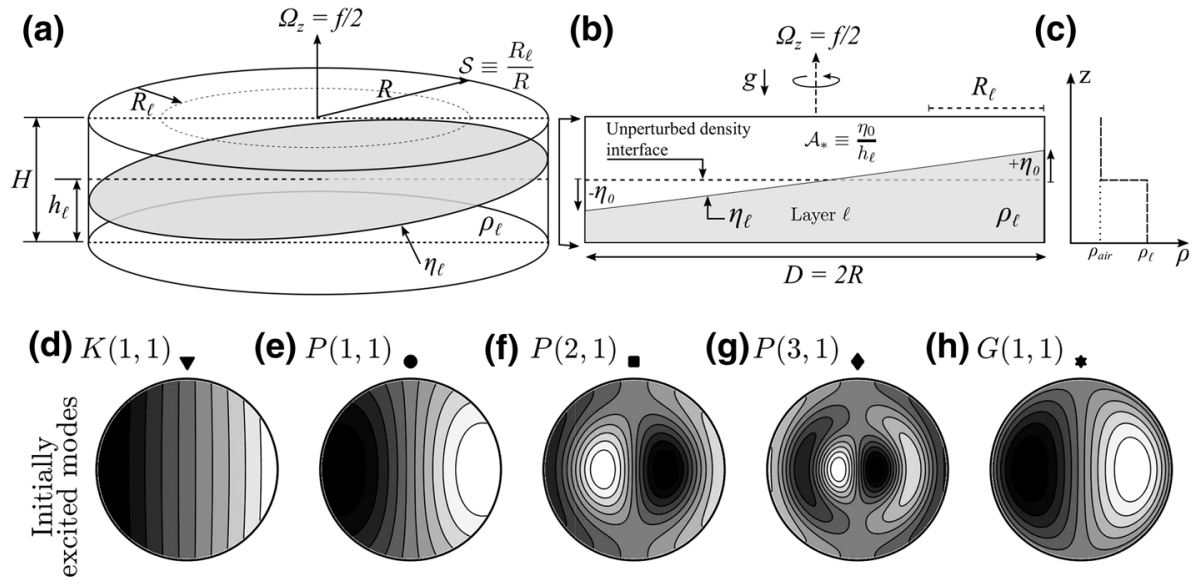

(e) $P(1,1)$.
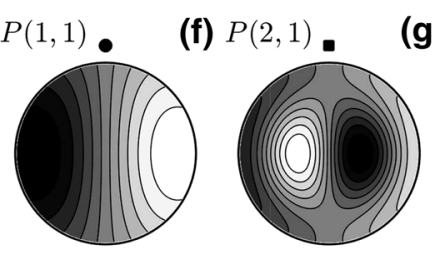

(g) $P(3,1)$

(h) $G(1,1)$ *

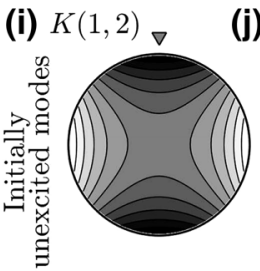

(j) $K(1,3)_{\nabla}$

(k) $P(1,2)$

(I) $P(1,3)$ 。
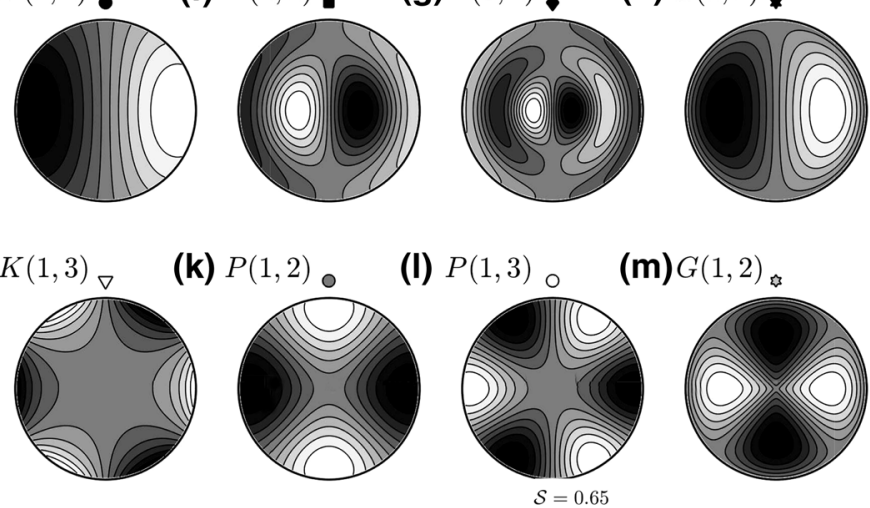

(m) $G(1,2)$

(n) $B(1,0)$

(o) $B(2,0)$

(p) $B(3,0)$
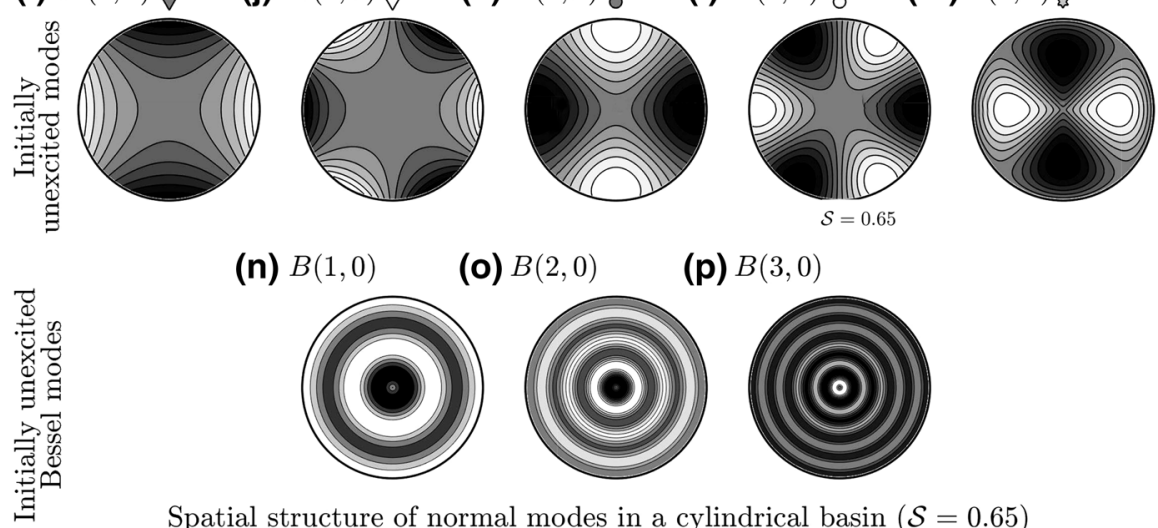

Spatial structure of normal modes in a cylindrical basin $(\mathcal{S}=0.65)$

$$
\frac{\eta_{\ell}(r, \theta)}{\eta_{\max }}
$$

$-1$

$$
-0.5
$$

0

0.5

Fig. 1 Upper panel. Schematic of the conceptual model: a rotating cylindrical basin of radius $R$ containing a fluid layer $h_{\ell}$ with density $\rho_{\ell} . S \equiv R_{\ell} / R$ is the Burger number, the ratio of the Rossby radius of deformation, $R_{\ell}$, to the basin's radius. b Two-dimensional plane across a diameter of the basin, $\eta_{\ell}$ denotes the density interface between the layer $\ell$ and the environmental fluid. $\mathcal{A}_{*} \equiv \eta_{0} / h_{\ell}$ is the perturbation parameter, the ratio between the initial maximum air-water interface displacement, $\eta_{0}$, and the unperturbed layer thickness, $h_{\ell}$. c Vertical density profile of the unperturbed density interface. The density of the air is considered negligible. Panel $\mathbf{d}-\mathbf{p}$ spatial structure of the density interface $\eta_{\ell} / \eta_{0}$ of the main initially excited modes $(\mathbf{d}-\mathbf{h})$ and unexcited modes $\mathbf{i}-\mathbf{p}$ for a Burger number $S=0.65$. Normal modes are classified regarding their radial and azimuthal wave numbers, $n_{r}$ and $n_{\theta}$, respectively: Kelvin modes, $K\left(n_{r}, n_{\theta}\right)$, Poincaré modes, $P\left(n_{r}, n_{\theta}\right)$, Geostrophic mode, $G\left(n_{r}, n_{\theta}\right)$, Bessel modes, $B\left(n_{r}, n_{\theta}=0\right)$. The initially excited modes are characterised by $n_{r} \geq 1$ and $n_{\theta}=1$

spectrum, $\omega / f \geq 10$ (compare green line with the blue and red lines in Fig. 4g). In the $\mathrm{M}-\mathrm{RR}$, the relative error between theoretical and experimental frequencies of the fundamental modes is $9 \%$. It is possible that the shifts in peak frequencies in Fig. 4, with respect to theoretical frequencies, correspond to nonlinear and viscous detuning [5, 26, 29, 31]. The analytical solution (2a) most likely also tells us something about the relative height/ strength of the excited modes, but we will not discuss it here. 

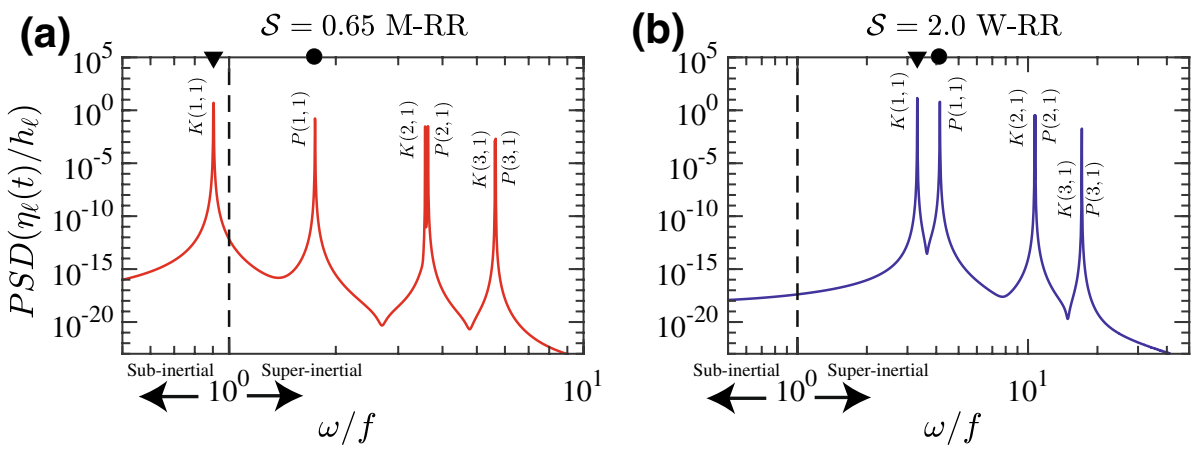

Fig. 2 Power spectra of theoretical time series of $\eta_{\ell}(t) / h_{\ell}$ resulting from Eq. (3a) for Burger number $S=0.65$ (M-RR) and $S=2.0$ (W-RR), respectively. The spectra identify the gravest Kelvin and Poincare wave frequencies, $K(1,1)$ and $P(1,1)$, along with the conjugate modes $K(2,1), P(2,1)$ and $K(3,1), P(3,1)$ forced by the initial condition. Dashed-line denotes the dimensionless inertial frequency

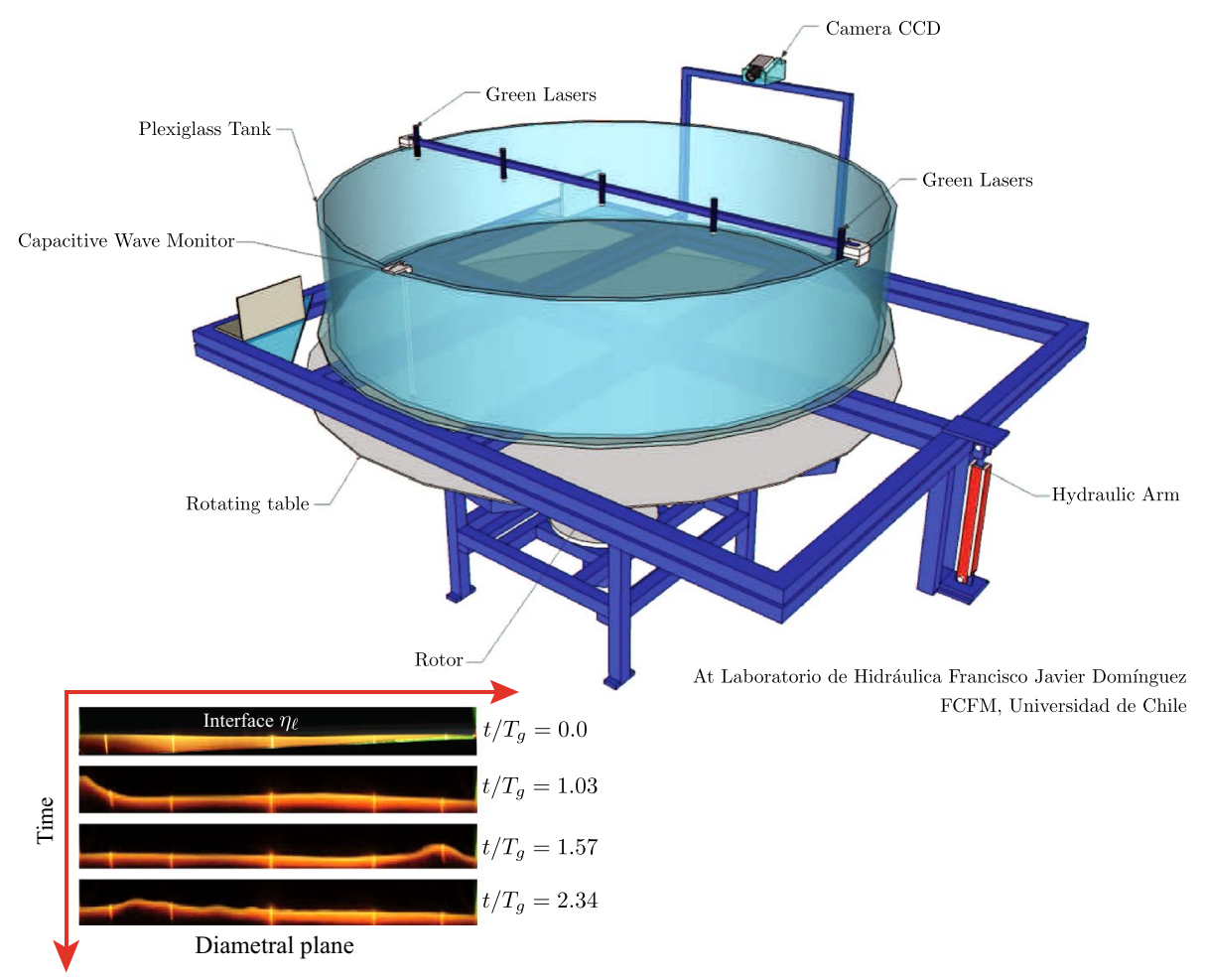

Fig. 3 Experimental facility: rotating table system. Examples of diametral planes recorded on the laserinduced optical fluorescence sheet. The air-water interface is found on the upper boundary of the orange colour 
Moderate-Rotating Regime (M-RR)

Weak-Rotating Regime (W-RR)
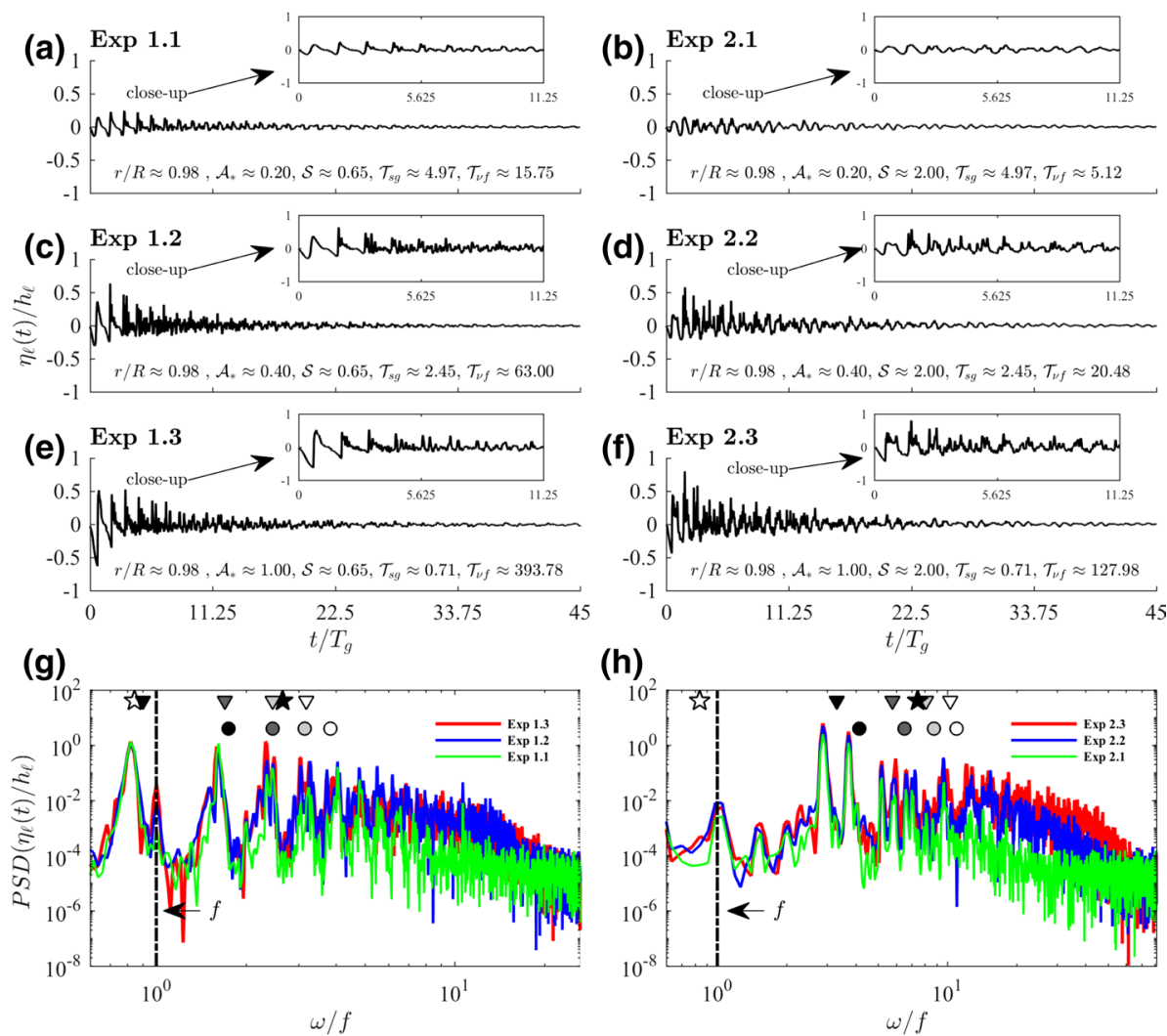

(h)

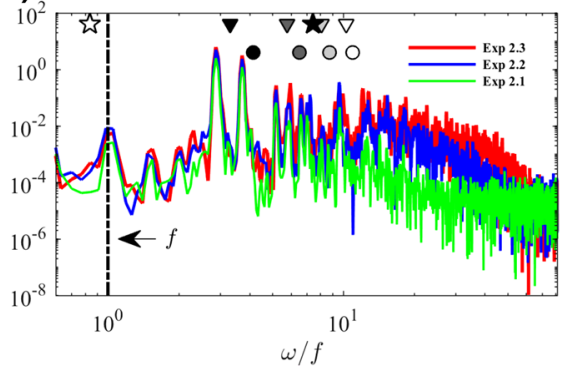

Fig. 4 a-f Time series of interface displacement, $\eta_{\ell}\left(t / T_{g}, r / R \approx 0.98, \theta=-\pi / 2\right)$. (g-h) Power spectra of $\eta_{\ell} / h_{\ell}$. Dash-dotted line: inertial frequency, $\omega / f=1$. Gravest Kelvin wave frequency $\left(\boldsymbol{\nabla}: \omega_{K(1,1)} / f\right)$, gravest Poincaré wave frequency $\left(\bullet: \omega_{P(1,1)} / f\right)$, and the Kelvin-Poincaré triad frequencies $\left(\star: \omega_{K P}^{+} / f=\left(\omega_{P(1,1)}+\omega_{K(1,1)}\right) / f\right.$, 饮: $\left.\omega_{K P}^{-} / f=\left(\omega_{P(1,1)}-\omega_{K(1,1)}\right) / f\right)$. Triangles and circles to the right of the gravest frequencies denote sub-azimuthal Kelvin and Poincaré modes, $K\left(n_{r}=1, n_{\theta}=2,3,4\right)$ and $P\left(n_{r}=1, n_{\theta}=2,3,4\right)$, respectively

We examine the temporal variability of the wavefield via the WS. Figure 5 shows the WS of $\eta_{\ell}(t) / h_{\ell}$ for the same subset of experiments. In the case of the M-RR (left panels), the WSs show that the energy in the wavefield is initially located in the gravest Kelvin and Poincaré waves (see $\boldsymbol{\nabla}$ and $\bullet$ markers). However, after the first period, a continuous range of high frequencies is strongly energised (see Fig. 5a-c). This process is observed every $2.4 T_{g}$, which corresponds to the normal mode period, $T_{K(1,1)}$. The results in Fig. 5a-c suggests that the periodicity of the high-frequency band is the spectral signature of a nonlinearly steepened wave train passing the observation spot once every $T_{K(1,1)}$.

It is found that both the gravest Kelvin and Poincare modes lose substantial energy over ten wave periods. In the case of the Kelvin mode, the WS shows a robust exponential decay structure as a function of time (see blue line in left sub-panels). In contrast, although the Poincaré wave energy has a similar but weaker exponential decay signature as a function of time, this also shows periodic increments of energy (see red line in left sub-panels). In the case of the M-RR, after four periods, most of the energy is contained in high frequencies. 

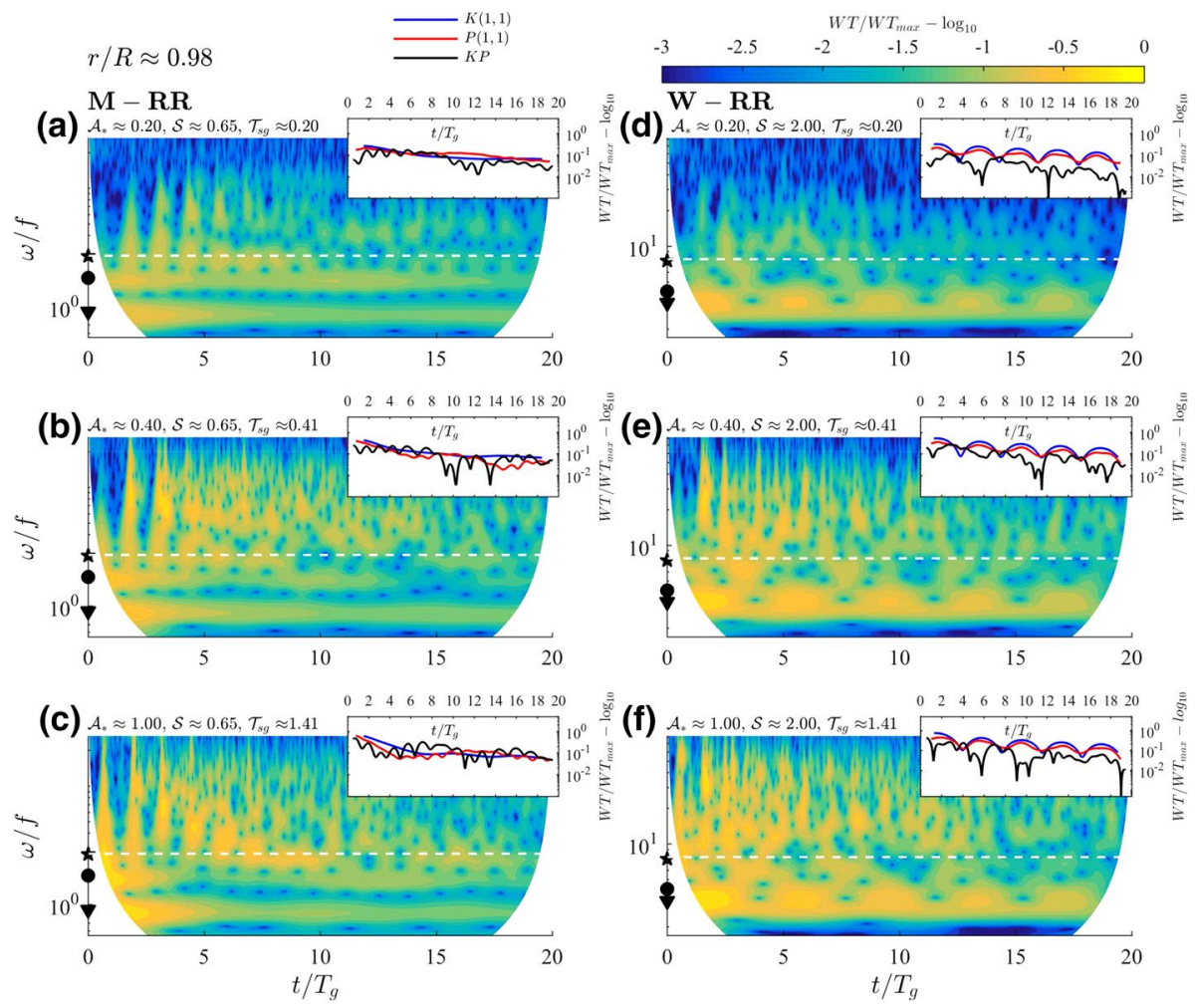

Fig. 5 Wavelet spectra of the interface displacement, $\eta_{\ell}\left(t / T_{g}, r / R \approx 0.98, \theta=-\pi / 2\right) / h_{\ell}$. Left panels show M-RR, $S=0.65$. Right panels show weak-rotational regimes, $S=2.0$. Initial perturbations are: a, d $\mathcal{A}_{*} \approx 0.2 ; \mathbf{b}, \mathbf{e} \mathcal{A}_{*} \approx 0.4 ; \mathbf{c}, \mathbf{f} \mathcal{A}_{*} \approx 1.0$. Subplots in the right-up corner on each panel show the temporal evolution of wavelet spectra at the Kelvin frequency $\left(\boldsymbol{\nabla}: \omega_{K(1,1)} / f\right)$, Poincaré frequency $\left(\bullet: \omega_{P(1,1)} / f\right)$, and the Kelvin-Poincaré frequency $\left(\star: \omega_{K P}^{+} / f=\left(\omega_{P(1,1)}+\omega_{K(1,1)}\right) / f\right)$, respectively. White-dashed line denotes the dimensionless frequency of the Bessel mode $B(1,0), \omega_{B, 0} / f$ (see Table 2)

In particular, the energy contained in the potential triad frequency $\omega_{K P}^{+}=\omega_{K(1,1)}+\omega_{P(1,1)}$ (see $\star$ marker) shows a periodical structure, remaining energised after ten wave periods. The back-and-forth of energy content in the frequency $\omega_{K P}^{+}$is attributed to the Poincare wave dynamics. Numerical studies $[16,47]$ have shown that the Poincaré wave can either transfer or receive energy from other modes which lead to a periodic increment of its energy contents. The gravest Kelvin wave, contrarily, only transfers its energy to higher modes. The degeneration processes of the Kelvin wave lead to a significant change in the wavefield structure, which is mostly characterised by sub-azimuthal modes $\left(K\left(n_{r}, n_{\theta}>1\right)\right.$ and a periodic signal associated with the Kelvin-Poincaré coupling.

In the W-RR, $S=2.0$, the near-lateral boundary wavefield shows substantial differences compared with the M-RR. In the case of Exp. 2.1 (Fig. 4b), the initial perturbation $\mathcal{A}_{*}=0.2$ leads to $\mathcal{T}_{s g} \approx 4.97 \leq \mathcal{T}_{v f} \approx 5.12$. For this set of parameters, the basin-scale waves do not show a clear steepening or dispersive process, and after the third wave period, it is not possible to distinguish a robust basin-scale wave signature. The increase of the initial perturbation, $\mathcal{A}_{*}=0.4$, leads to a substantial separation between the steepening parameters, $\mathcal{T}_{s g} \approx 2.45$, and the inverse Ekman parameter, $\mathcal{T}_{v f} \approx 20.48$. In this scenario (see Exp. 2.2 in Fig. 4d), 
we observe steepening and wave dispersion during the second seiche period. However, the wave-packet structure is different to the solitary-type train of waves found in the M-RR, where the amplitude of each solitary-type wave is smaller than the previous. In the W-RR, there is a second period characterised by the passing of a wave-packet, but this structure is not observed anymore after the third period. The time series of Exp. 2.3 (Fig. 4f) is characterised by $\mathcal{A}_{*}=1.0$, and $\mathcal{T}_{s g} \approx 0.71 \leq \mathcal{T}_{v f} \approx 127.98$. Similar to Exp. 2.2, Exp. 2.3 shows a strong steepening and dispersion of the basin-scale wave leading to a wave-packet during the first two periods. The structure of this wave-packet has a strong similarity to the ones observed in a two-layer non-rotating channel $[9,25]$. However, unlike earlier experiments in non-rotating systems, here the wave-packet pattern is barely identified in later seiche periods.

Figure $4 \mathrm{~h}$ shows the PSD of $\eta_{\ell}(t) / h_{\ell}$ in the W-RR. In this rotational regime, the wavefields concentrate the spectral energy in the super-inertial frequency band, with robust peaks close to the gravest Kelvin and Poincaré modes and at the inertial frequency (dot-dashed line). Similar to the M-RR, the energy of the wavefield increases as $\mathcal{A}_{*}$ increases, especially in the high-frequency range, $\omega / f \geq 10$. In the $\mathrm{W}-\mathrm{RR}$, the relative error between theoretical and experimental frequencies of the fundamental modes is $11 \%$ which is larger than the deviation observed in the M-RR.

Figure 5 (right panels) shows the temporal variability of spectral energy in the W-RR. We observe that the gravest Kelvin and Poincaré frequencies have recurrent increments of energy in phase, approximately every $3.75 T_{g} \approx 19.38 \mathrm{~s}$ approximately. This period matches with the period associated with the triad $\omega_{K P}^{-}=\omega_{P(1,1)}-\omega_{K(1,1)}, T_{K P}^{-}=19.39 \mathrm{~s}$, suggesting that the waves can be found in phase every $T_{K P}^{-}$. Additionally, the spectral energy in the high-frequency band shows a significant variability as a function of time. After ten wave periods, most of the remaining energy is contained in high-frequency modes. This temporal evolution in the spectrum suggests that there is a significant transfer of energy from large to smaller scales controlled by the degeneration of the basin-scale gravity waves in lateral boundary regions. Similar to the M-RR, the potential triad Kelvin-Poincaré frequency, $\omega_{K P}^{+}$, is periodically energised but it remains with lower energy than the parent Kelvin and Poincaré modes (see $\star$ marker in right sub-panels in Fig. 5). We stress that when the parent frequencies $\left(\omega_{K(1,1)}\right.$ and $\left.\omega_{P(1,1)}\right)$ reach an energy peak, the frequency $\omega_{K P}^{+}$shows a substantial drop of energy (compare the red and blue lines with the black line in sub-panels of Fig. 5d-f). However, the nature of these signals is not evident.

\subsection{Interior gravity wavefield}

We examine the gravity wavefield in two different interior zones. The first position is located at $r / R \approx 0.5$, making sure the signature of trapped boundary Kelvin waves are found in the spectral wavefield. The second location is at the centre of the basin, $r / R \approx 0$, and therefore a pivot point for the basin-scale waves. This position was chosen since we expect a significantly different wavefield than the observed one at the near-lateral boundary but also because previous authors [16] have identified via numerical experiments the energisation of a persistent standing wave of frequency $\omega_{K P}^{+}$.

\subsubsection{Wavefield at $r / R \approx 0.5$}

Figure 6 shows the interface response, $\eta_{\ell} / h_{\ell}$ and the PSD for the same subset of experiments examined at the near-lateral boundary but now at $r / R \approx 0.50$; results for M-RR and $\mathrm{W}-\mathrm{RR}$ are shown on the left and right panels, respectively. 


\section{M-RR}
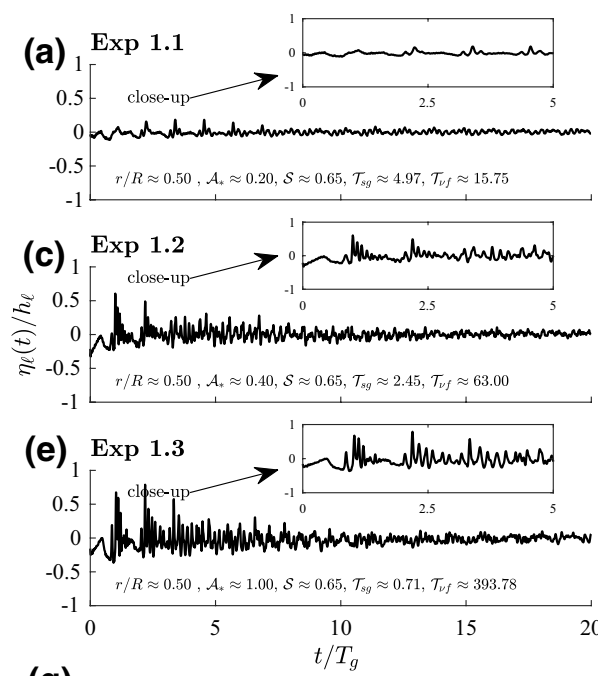

(g)

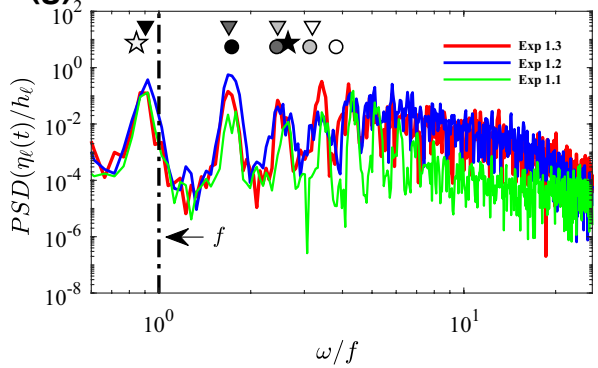

W-RR
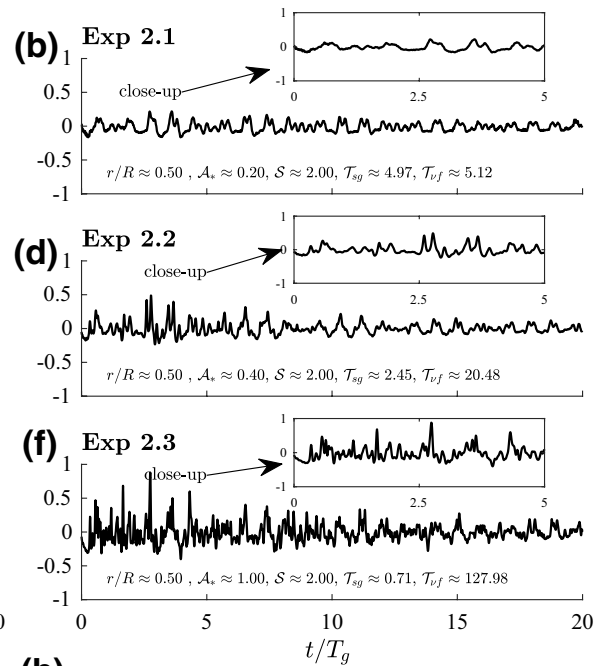

(h)

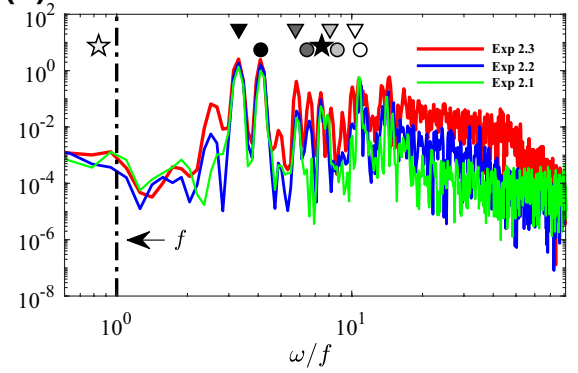

Fig. 6 a-f Time series of interface displacement, $\eta_{\ell}\left(t / T_{g}, r / R \approx 0.50, \theta=-\pi / 2\right) / h_{\ell}$. $\mathbf{g}-\mathbf{h}$ Power spectra of $\eta_{\ell} / h_{\ell}$. Legends idem to Fig. 4

In the case of the M-RR, although the structure of trains of waves along the time series is also observed, the wave-packet is different from the ones found at the near-lateral boundary. In Fig. 6a, the train of waves associated with $\mathcal{A}_{*}=0.2$ shows a beating-like wave pattern similar to the wave-packet found by Grimshaw and Helfrich [20] and Grimshaw et al. [24]. This beating wave structure remains in the interface signal for roughly seven seiche periods, and then its pattern degenerates to a high-frequency wavefield structure, along with a weak low-frequency signature. Figure $6 \mathrm{c}$, e show results for higher initial perturbations, $\mathcal{A}_{*}=0.4$ and $\mathcal{A}_{*}=1.0$; besides the larger wave amplitudes observed in $\eta_{\ell} / h_{\ell}$, the wave pattern is remarkably similar. However, unlike the first case $\mathcal{A}_{*}=0.2$, the beating wave packages and their envelope are geometrically skewed, similar to the solitary-type wave trains registered at the near-lateral boundary.

The PSD of the wavefield at $r / R \approx 0.5$ shows that the theoretical basin-scale Kelvin and Poincaré frequencies, $\omega_{K(1,1)}$ and $\omega_{P(1,1)}$, are still associated with the largest energy peaks. Nevertheless, in this zone, we observe that the energy peak associated with the gravest Poincaré mode, $P(1,1)$, might also be linked to the second-azimuthal Kelvin wave frequency, $\omega_{K(1,2)}$. Similarly, the higher azimuthal Kelvin and Poincaré frequencies are indistinguishable from each other in the spectra. However, we expect that Poincaré modes 
contribute substantially to the energy peaks found in interior regions given their spatial modal amplitude (see lower panels in Fig. 1). In this zone, the relative error between theoretical and experimental frequencies of $K(1,1)$ and $P(1,1)$ is $3 \%$, which is significantly lower than the relative error estimated at the near-lateral boundary.

Figure 7 (left panels) shows the spectral energy evolution of the wavefield in the M-RR. Figure 7a shows the WS of the beating wave-like pattern illustrated in Fig. 6a. In this case, the most energetic frequencies are associated with the gravest Kelvin and Poincaré modes along with a pulsatile energy signal associated with higher frequencies $(4<\omega / f<7)$. This pulsating signal has roughly the period of the gravest Kelvin wave, $T_{K(1,1)}$. The spectral energy of $K(1,1)$ and $P(1,1)$ decays significantly after eight seiche periods, and the residual energy is mostly stored at high frequencies. In the case of experiments with larger initial perturbations (see Fig. 7b, c), there is a substantial amount of energy at high frequencies relative to the energy stored in the gravest modes. However, the high-frequency spectrum shows a strong decay after six wave periods.

In the case of the W-RR (see right panels in Fig. 6), we found a complex wavefield structure with no regular wave pattern. However, it is observed that two merged waves modulate the evolution of the interface displacement. This behaviour is observed in Fig. 6b, d, especially after ten seiche periods in Exp. 2.2, and almost along the full time series in Exp. 2.1. Weaker rotational regimes lead to $K(1,1)$ and $P(1,1)$ becoming closer in
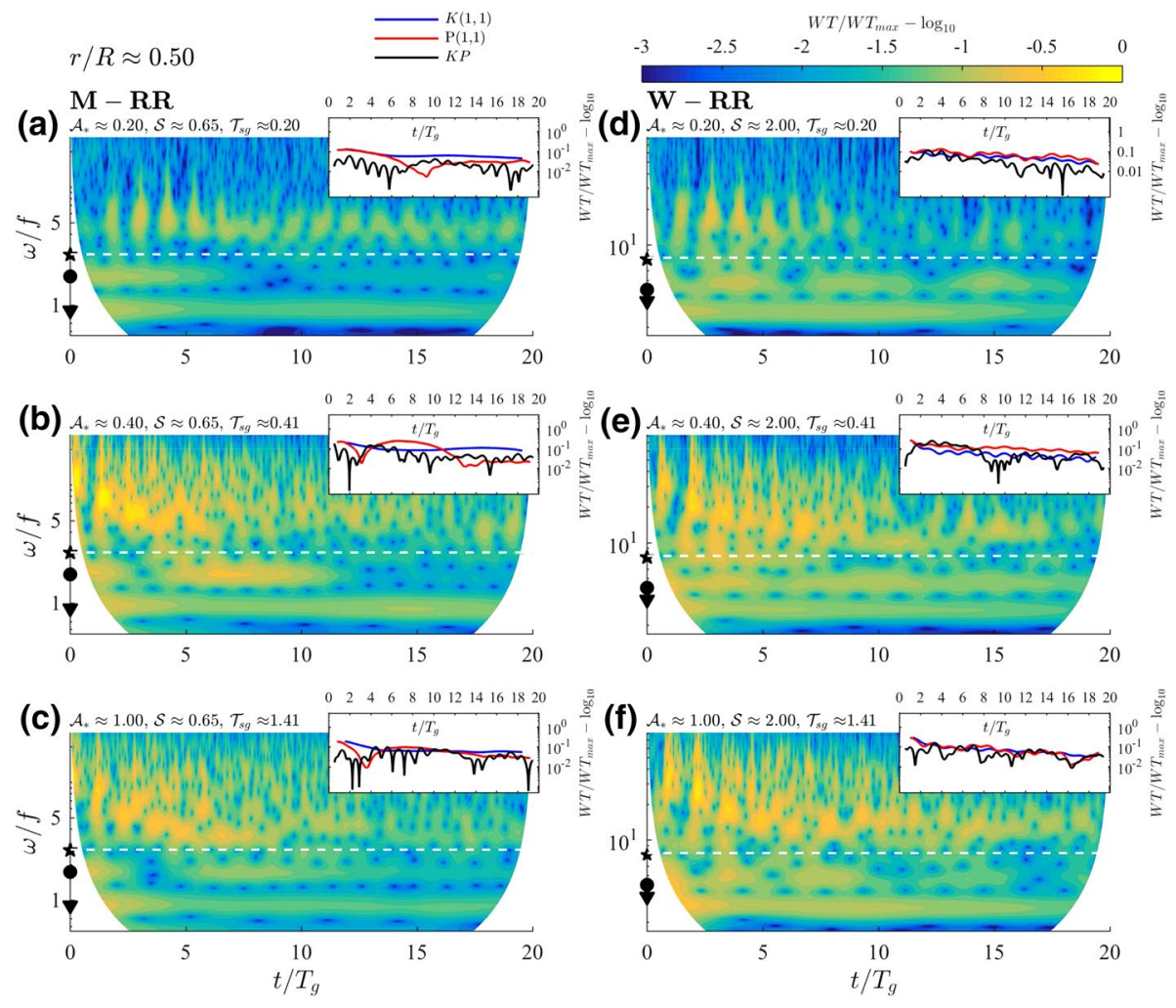

Fig. 7 Wavelet spectra of the interface displacement, $\eta_{\ell}\left(t / T_{g}, r / R \approx 0.50, \theta=-\pi / 2\right) / h_{\ell}$. Legends idem to Fig. 5 
frequency $[19,43]$. The two-wave signal pattern is associated with the fundamental superinertial Kelvin and Poincaré modes which have the highest spectral energy peaks in the PSD (see Fig. 6h). In this region, the relative error between theoretical and experimental frequencies of $K(1,1)$ and $P(1,1)$ is approximately $1 \%$, which is significantly lower than the relative error found in the near-lateral boundary wavefield.

Figure 7 (right panels) shows the temporal evolution spectral energy in the W-RR. We observe that the energy stored in the gravest Kelvin and Poincaré modes has a weak but detectable periodic structure. This pulsating signal suggests that both waves might be transferring and gaining energy either from each other or other higher modes. After the second seiche wave period, a substantial part of the spectral energy is found in frequencies greater than $\omega / f \sim O\left(10^{1}\right)$.

\subsubsection{Wavefield at $r / R \approx 0$}

Figure 8 shows the wavefield at the centre of the basin, $r / R \approx 0$, for the same subset of experiments. In the case of the M-RR, (left panels in Fig. 8), the interface displacement, $\eta_{\ell}(t) / h_{\ell}$, shows small wave amplitudes even for the largest initial perturbation parameter, $\mathcal{A}_{*}=1.0$. In Fig. $8 \mathrm{a}\left(\mathcal{A}_{*}=0.2\right), \eta_{\ell}(t) / h_{\ell}$ is well-characterised by a weakly damped periodic wave signal whose frequency is greater than that of the basin-scale frequency modes. As the initial perturbation parameter is incremented $\left(\mathcal{A}_{*}=0.2,0.4\right.$, see Fig. $\left.8 \mathrm{c}, \mathrm{e}\right)$, higher frequencies constitute the temporal structure of $\eta_{\ell}(t) / h_{\ell}$ (compare close-up panels in Fig. 8a, c, e). The fact that the maximum amplitudes reached by $\eta_{\ell}(t) / h_{\ell}$ are similar, independent of the value of $\mathcal{A}_{*}$, indicates that the centre of the basin is a pivot point for basin-scale waves. This result also suggests that most of the energy remains near the lateral boundary region even for the M-RR. Indeed, in the PSD of $\eta_{\ell}(t) / h_{\ell}$ (see Fig. 8g), we found no energy peaks associated with basin-scale waves. Instead, we found energy peaks associated with the potential triad frequency $\omega_{K P}^{+}(\star)$ and the purely radial mode frequencies, here denoted by $\omega_{B\left(n_{r}, 0\right)}$ (dashed lines). In the case of the first Bessel mode, $B(1,0)$, its theoretical frequency, $\omega_{B(1,0)}$, is significantly close to $\omega_{K P}^{+}$[53]. de la Fuente et al. [16] observed via numerical experiments that nonlinear dynamics of Kelvin waves were associated with the emerging of a standing-like wave in the centre of the basin with frequency $\omega_{K P}^{+}$. Moreover, Ulloa et al. [53] found via laboratory experiments that the frequency $\omega_{B(1,0)}$ has a spectral signature in the wavefield at the lateral boundaries as well, with a magnitude proportional to the intensity of nonlinearities. Here, the highest spectral energy peaks in Fig. $8 \mathrm{~g}$ are not associated with the initially energised wave base field; therefore, they have to be excited via nonlinear dynamics of the wavefield itself. Additionally, the WS of $\eta_{\ell}(t) / h_{\ell}$ (see left panels of Fig. 9) also show that spectral energy is mostly stored at $\omega_{K P}^{+}$and $\omega_{B(1,0)}$. However, as the initial perturbation is increased, frequencies close to the Bessel modal frequencies $\omega_{B(2,0)}$ and $\omega_{B(3,0)}$ are also found energised (see dashed lines in Fig. 8g, h).

In the case of W-RR, there is a significant change in the wavefield pattern at $r / R \approx 0$ (see right panels in Fig. 8). First, we found larger wave amplitudes and complex dynamics, including long-frequency envelope wave patterns for the weak initial perturbation case (see Fig. $8 \mathrm{~b}, \mathcal{A}_{*}=0.2$ ). Similar to the M-RR case, the PSD shows a wavefield characterised by high frequencies and negligible signals associated with basin-scale frequencies. In particular, the WS of $\eta_{\ell}(t) / h_{\ell}$ (see right panels in Fig. 9) show that energy remains stored at $\omega_{K P}^{+}$and $\omega_{B(1,0)}$ during the first ten seiche periods, with significant energy fluctuations as a function of time in high-frequency ranges $\omega / f>10$. However, it is unclear how a narrow range of frequencies around $\omega_{K P}^{+}$and $\omega_{B(1,0)}$ can be energised. Initially, and while nonlinear 
M-RR
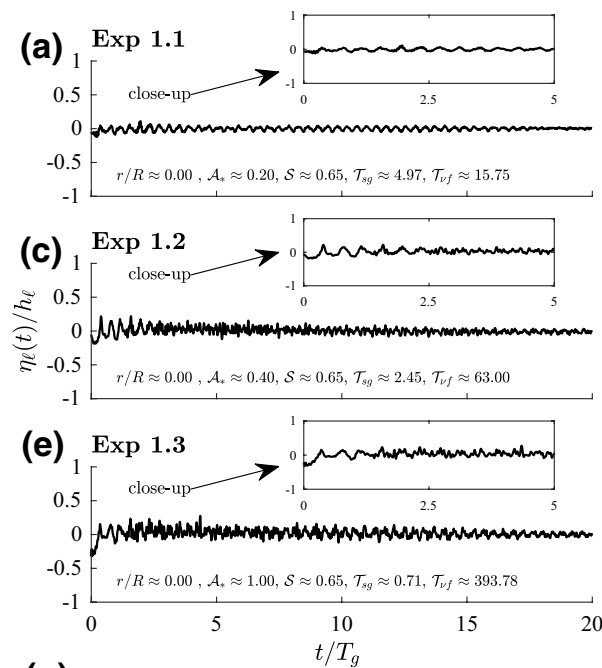

(g)

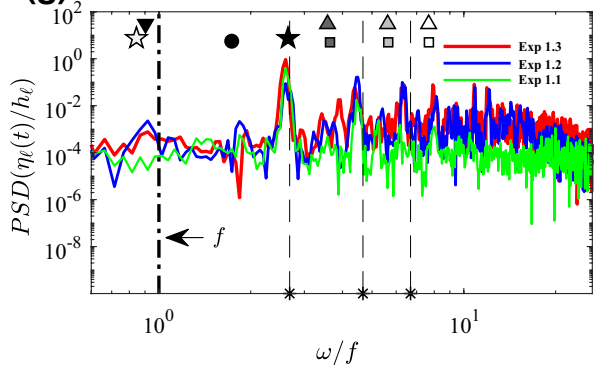

W-RR
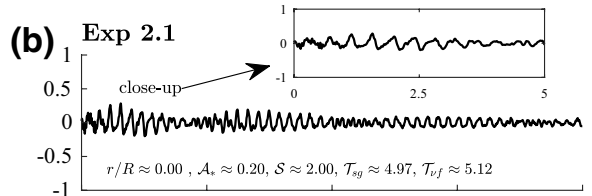

(d)
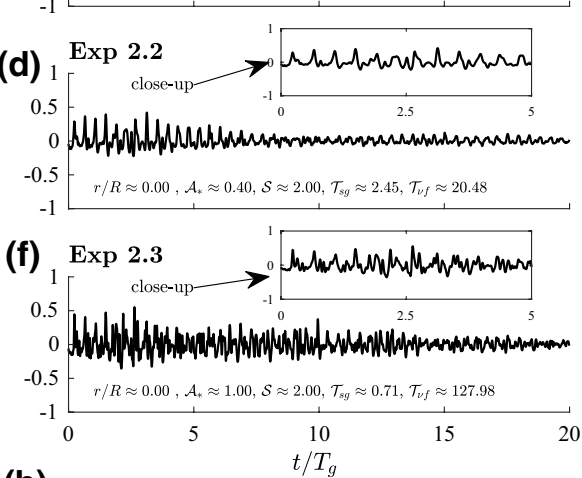

(h)

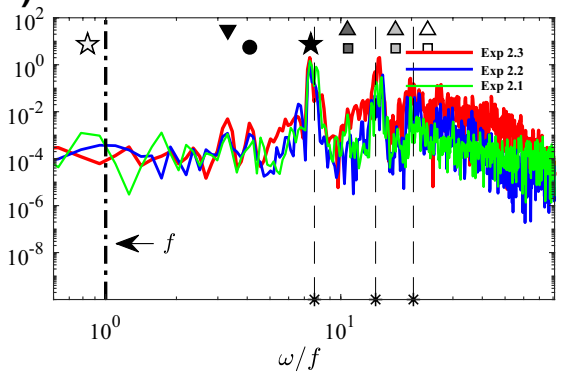

Fig. 8 a-f Time series of interface displacement, $\eta_{\ell}\left(t / T_{g}, r / R \approx 0, \theta=-\pi / 2\right) / h_{\ell}$. $\mathbf{g}$-h Power spectra of $\eta_{\ell} / h_{\ell}$. Kelvin wave frequency $\left(\nabla: \omega_{K(1,1)} / f\right)$, Poincaré wave frequency $\left(\bullet: \omega_{P(1,1)} / f\right)$, and the KelvinPoincaré triad frequencies $\left(\star: \omega_{K P}^{+} / f=\left(\omega_{P(1,1)}+\omega_{K(1,1)}\right) / f\right.$, $\left\langle: \omega_{K P}^{-} / f=\left(\omega_{P(1,1)}-\omega_{K(1,1)}\right) / f\right)$. Triangle and squares to the right of the fundamental frequencies denote sub-radial Kelvin and Poincaré modes, $K\left(n_{r}=2,3,4, n_{\theta}=1\right)$ and $P\left(n_{r}=2,3,4, n_{\theta}=1\right)$, respectively. Dashed lines denote the frequencies of the first three Bessel modes, $\omega_{B}\left(n_{r}=1,2,3, n_{\theta}=0\right)$

dynamics stay strong, a potential triad nonlinear coupling between the gravest Kelvin and Poincaré waves can store energy in $\omega_{K P}^{+}$and energise the natural modal frequency $\omega_{B(1,0)}$. yet, near $r / R \approx 0$, the modes $K(1,1)$ and $P(1,1)$ have practically no energy content. Therefore, if a Kelvin-Poincare coupling is forcing this range of frequencies, energy should be radiated from the near-lateral boundary $(r / R \approx 1)$ toward the interior.

\subsection{Decay rate of basin-scale waves}

We examine the bulk decay rate of the basin-scale waves in the near-lateral boundary region, where most of the initial potential energy is available for energising gravity waves. Here, we compute the $e$-folding bulk damping rate of the wavefield based on the absolute magnitude of the interface displacement, $\left|\eta_{\ell}(t)\right| / h_{\ell}$ (see example in Fig. 10a). The decay of $\left|\eta_{\ell}(t)\right| / h_{\ell}$ is modelled by $\mathcal{A}_{*} \exp \left(-\mathcal{T}_{d g}^{-1} t / T_{g}\right)$, and for each experiment we 

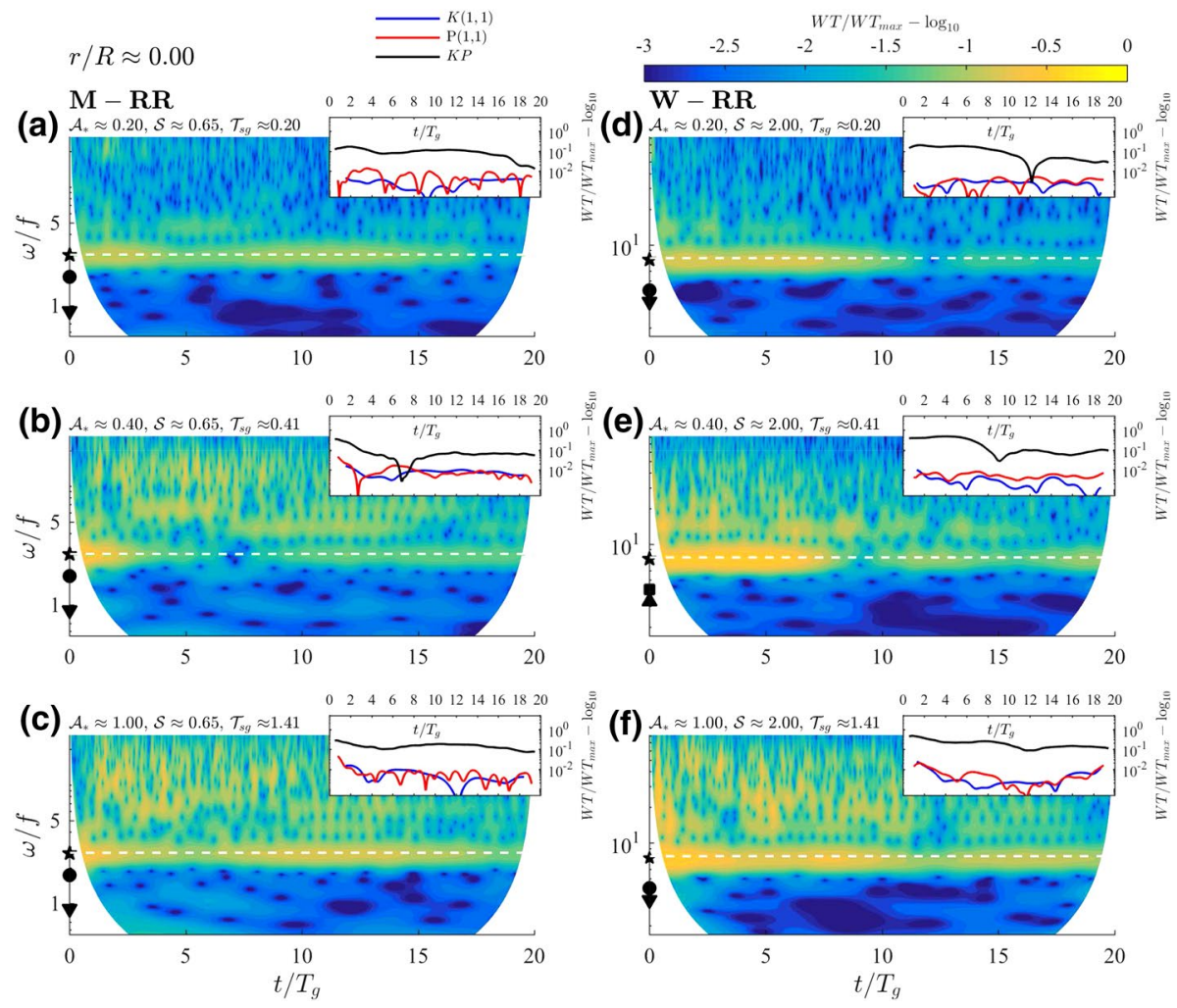

Fig. 9 Wavelet spectra of the interface displacement, $\eta_{\ell}\left(t / T_{g}, r / R \approx 0.0, \theta=-\pi / 2\right) / h_{\ell}$. Legends idem to Fig. 5

fit the exponential model to find the bulk damping rate of the wavefield, $\mathcal{T}_{d g}^{-1} \equiv T_{g} / T_{d}$, where $T_{d}$ is the dimensional damping timescale.

In Fig. 10b-e, we summarise the computed damping rates, $\mathcal{T}_{d g}^{-1}$, as a function of the dimensionless parameters $\mathcal{A}_{*}, S, \mathcal{T}_{s g}$, and $\mathcal{T}_{v f}$. Our findings are the following: (1) the higher initial perturbation $\mathcal{A}_{*}$, the higher the bulk damping rate, $\mathcal{T}_{d g}^{-1}$ (see Fig. 10b); (2) the stronger and faster the nonlinear dynamics start affecting basin-scale waves, the stronger the bulk damping rate, $\mathcal{T}_{d f}$ (see Fig. 10d); (3) the weaker the effect of viscosity relative to rotating effects, the higher the bulk decay rate, $\mathcal{T}_{d g}^{-1}$ (see Fig. 10e); and (4) although the data do not show a robust tendency, we observed that stronger bulk decay rates, $\mathcal{T}_{d g}^{-1}$, are associated with stronger rotational regimes (see Fig. 10c).

Figure 10f shows the best fitting for $\mathcal{T}_{d g}^{-1}$ in terms of a nonlinear combination of the Burger number, $\mathcal{S}$, the steepening parameter, $\mathcal{T}_{s g}$, and the inverse Ekman parameter, $\mathcal{T}_{v f}$, $\mathcal{T}_{d g}^{-1}=c_{0}(S)^{c_{1}}\left(\mathcal{T}_{s g}\right)^{c_{2}}\left(\mathcal{T}_{v f}\right)^{c_{3}}$, with $c_{i}(i=0,1,2,3)$ constant coefficients to be solved by using a least square minimisation. We obtained $c_{0}=0.0014, c_{1}=0.2763, c_{2}=0.9340$, and $c_{3}=0.8518$. Hence, the experimental fitting of the $e$-folding damping timescale shows that $\mathcal{T}_{d g} \sim T_{g}^{1.2} T_{f}^{0.58} T_{v}^{-0.85} T_{s}^{-0.93}$. The sign of the exponents of the relevant timescales lead to the same findings drawn above. We stress that the steepening timescale and the inertial period are inversely proportional to the damping rate of the basin-scale waves. 

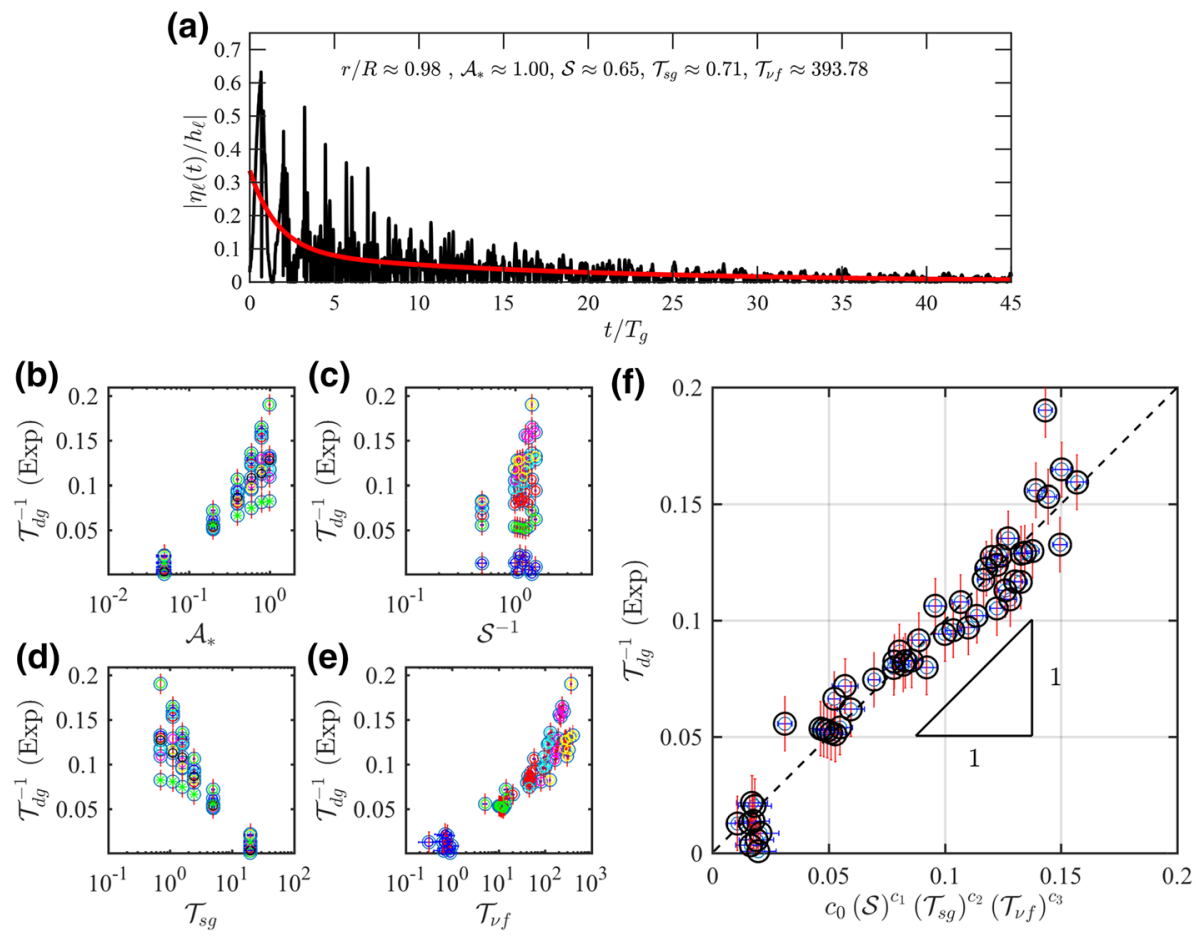

Fig. 10 a Example of the absolute dimensionless interface displacement, $\left|\eta_{\ell}(t)\right| / h_{\ell}$, as a function of time. Experimental decay rate, $\mathcal{T}_{d g}^{-1}$, as a function of $\mathbf{b}$ initial perturbation $\mathcal{A}_{*}$, $\mathbf{c}$ Burger number $S$, $\mathbf{d}$ steepening parameter $\mathcal{T}_{s g}$, and $\mathbf{e}$ inverse Ekman parameter $\mathcal{T}_{v f}$. f Data fit for decay rate as a function of the dimensionless parameters; dashed-line indicates the identity

\section{Discussion and conclusions}

In this study, we have examined the gravity wavefield in weak-rotational environments via laboratory experiments in a rotating table. Note that if we neglect diffusion processes, our experiments are analogous to the first vertical mode dynamics of a two-layer system with a surface rigid-lid hypothesis. Different authors $[18,35,50]$ have investigated and formulated the dynamics of nonlinear waves in rotating systems by using an equivalent singlelayer problem in terms of a set of coupled equations. Thus, the experiments can represent the wavefield dynamics of the first vertical mode in a rotating and density-stratified fluid, obviating diffusion processes associated with miscible stratified fluids. Controlling the initial perturbation of the interface $\eta_{\ell}$ and the background inertial frequency, $f$, in the basin, we have found a significant spatiotemporal variability of the gravity wavefield evolution across the radial position.

In the strongest rotational regime here investigated (M-RR, $S=0.65$ ), the gravity wavefield observed in the near-lateral boundary, $r / R \approx 0.98$, is similar to the internal gravity wavefield observed by Ulloa et al. [53], characterised by a strong nonlinear degeneration of the basin-scale waves into solitary-type wave trains as the initial perturbation, $\mathcal{A}_{*}$, was increased. In the M-RR, both nonhydrostatic and rotating-induced dispersion control the decay of the gravest Kelvin wave [21-23] and the delay of solitary wave formation [20, 
24]. In interior regions, $r / R \approx 0.50$, far from lateral boundaries, the basin-scale gravity waves degenerated into a beating-like wave pattern similar to the wave-packet found by Grimshaw et al. [24]. In the centre of the basin, the wavefield was dominated by a standinglike oscillation observed via numerical experiments by de la Fuente et al. [16], whose frequency matches with the resonant triad $\omega_{K P}^{+}=\omega_{K(1,1)}+\omega_{P(1,1)}$ and the Bessel mode, $\omega_{B(1,0)}$.

In the weakest rotational regime here investigated (W-RR), the gravity wavefield was characterised by a super-inertial frequency regime. In this regime, the gravest Kelvin and Poincaré waves have similar spatiotemporal structures, and both modes exhibit loss and gain of energy as a function of time. The transfer of energy towards the gravest Kelvin wave from higher modes has not been reported in sub-inertial frequency regimes, $\omega_{K(1,1)} / f<1$ $[16,47]$, associated with strong to moderate rotational environments, $S \leq 1 / \sqrt{2}$. Therefore, our results show that the W-RR can radically change the behaviour of gravest cyclonic Kelvin wave. Similar to the M-RR, the wavefield in the centre of the basin is strongly modulated by a standing-like wave with frequency $\omega_{B(1,0)}$ which matches $\omega_{K P}^{+}$, but its wavefield shows an amplitude significantly larger than the one observed in the M-RR.

Since the Bessel mode $B(1,0)$ is not initially excited by the initial value problem, stored energy near the frequency $\omega_{B(1,0)}$ should be the result of an energy transfer. Although it is not evident the energisation process, nonlinear wave dynamics and a possible Kelvin-Poincaré coupling in the near-lateral boundary are possible mechanisms to transfer energy from this region toward the interior and to the specific frequency $\omega_{B(1,0)}$.

As a general observation, the relative error of the theoretical Kelvin and Poincare wave frequencies derived from the linear dispersion relation[14, 49] decreases from the lateral boundary to the interior. The substantial mismatch between the theoretical linear frequencies and the experimental results might be related with nonlinear resonant interactions [51] and viscous detuning [5, 26, 29, 31]. Therefore, one might expect that the basin-scale wavefield in regions closer to the lateral boundary is more susceptible to viscous and nonlinear detuning, since it is subject to stronger nonlinearities (steepening, nonhydrostatic and rotating-induced dispersion) than the interior wavefield. Similarly, as the effect of nonlinearities and the lateral wall decreases towards the interior, we might expect that linear theory might better predict the experimental frequencies of the interior gravity wavefield.

The decay timescale computed from our experiments can be written as a function of the external parameters:

$$
\mathcal{T}_{d g} \approx 1.4 \times 10^{-3}\left(\frac{2 \pi R}{c_{\ell}}\right)^{0.27}\left(\frac{2 \pi}{f}\right)^{0.58}\left(\frac{\eta_{0}^{2}}{v}\right)^{-0.85}\left(\sqrt{1+\frac{\eta_{0}}{h_{\ell}}}-\sqrt{1-\frac{\eta_{0}}{h_{\ell}}}\right)^{0.93} .
$$

Therefore, we found that the damping timescale of the basin-scale gravity waves is proportional to the strength of background rotation, $f$, the basin length-scale, $R$, and the initial perturbation amplitude, $\eta_{0}$, and inversely proportional to the phase speed celerity, $c_{\ell}$. From the laboratory results in weak-rotational environments, we show that nonlinear mechanisms play a fundamental role in the decay rate of the gravity wavefield in near-lateral boundary zones as well as the regime of the background rotation. In interior zones, in contrast, the exponential decay model is not precisely suitable, as is shown in Figs. 6 and 8, especially in weak-rotational regimes $S \rightarrow 2$. One could argue that the evolution and decay structure of the interior wavefield is strongly modulated by Poincare waves which can receive and transfer energy, leading to a recursive growth and decay of wave amplitude reported by 
[16] and [47]. To quantify the decay rate of the interior gravity wavefield, a more complex model is required that accounts for the recursive temporal nature of Poincaré waves.

The rotational regime here explored is relevant for basin-scale hydrodynamic processes in mid- to small-size lake systems. One can expect similar gravity wavefields in systems such as Lake Kinneret (Israel) during mid summer conditions [3], Mono Lake (California) [32], Lakes Sempach, Baldegg and Hallwil (Switzerland) [7, 34] and the Araucanian lakes (Chile) [12].

Acknowledgements The authors thank the support of the Department of Civil Engineering of the University of Chile and the project Fondecyt No. 1140767. Hugo N. Ulloa thanks the support of CONICYT through its National Doctoral Scholarship No. 21110069 and the Physics of Aquatic Systems Laboratory (APHYS), École Polytechnique Fédérale de Lausanne, Switzerland. We also thank Alberto de la Fuente for his suggestions on this work and Tomás Trewhela who kindly read the manuscript and provided valuable comments during the revision process. Finally, we thank Peter Davies and the three anonymous referees for their critical review, insightful observations and helpful suggestions.

\section{References}

1. Abramowitz M, Stegun A (1965) Handbook of mathematical functions: with formulas, graphs, and mathematical tables. Dover, New York

2. Antenucci JP, Imberger J (2001) Energetics of long internal gravity waves in large lakes. Limnol Oceanogr 46(7):1760-1773. https://doi.org/10.4319/lo.2001.46.7.1760

3. Antenucci JP, Imberger J (2003) The seasonal evolution of wind/internal wave resonance in lake kinneret. Limnol Oceanogr 48(5):2055-2061. https://doi.org/10.4319/lo.2003.48.5.2055

4. Becherer JK, Umlauf L (2011) Boundary mixing in lakes: 1 Modeling the effect of shear-induced convection. J Geophys Res. https://doi.org/10.1029/2011JC007119

5. Belonozhko DF, Grigor'ev AI (2006) On internal nonlinear resonant interaction of capillary-gravitational waves on the flat surface of a viscous liquid. Tech Phys 51(12):1563-1575. https://doi. org $/ 10.1134 /$ S1063784206120036

6. Bendat J, Piersol A (2000) Random data. Wiley, New York. https://doi.org/10.1002/9781118032428

7. Beutel MW, Horne AJ (1999) A review of the effects of hypolimnetic oxygenation on lake and reservoir water quality. Lake Reserv Manag 15(4):285-297. https://doi.org/10.1080/07438149909354124

8. Birchfield GE (1969) Response of a circular model great lake to a suddenly imposed wind stress. J Geophys Res 74(23):5547-5554. https://doi.org/10.1029/JC074i023p05547

9. Boegman L, Ivey GN, Imberger J (2005) The energetics of large-scale internal wave degeneration in lakes. J Fluid Mech 531:159-180. https://doi.org/10.1017/S0022112005003915

10. Bouffard D, Boegman L, Rao YR (2012) Poincaré wave-induced mixing in a large lake. Limnol Oceanogr 57(4):1201-1216. https://doi.org/10.4319/lo.2012.57.4.1201

11. Bouffard D, Lemmin U (2013) Kelvin waves in lake geneva. J Great Lakes Res 39(4):637-645. https:// doi.org/10.1016/j.jglr.2013.09.005

12. Campos H (1984) Limnological study of Araucanian lakes (Chile). SIL Proc 1922-2010 22(2):13191327. https://doi.org/10.1080/03680770.1983.11897493

13. Cimatoribus AA, Lemmin U, Bouffard D, Barry DA (2018) Nonlinear dynamics of the nearshaore boundary layer of a large lake (Lake Geneva). J Geophys Res Oceans. https://doi.org/10.1002/2017J C013531

14. Csanady GT (1967) Large-scale motion in the great lakes. J Geophys Res 72(16):4151-4162. https:// doi.org/10.1029/JZ072i016p04151

15. Csanady GT (1968) Motions in a model great lake due to a suddenly imposed wind. J Geophys Res 73(20):6435-6447. https://doi.org/10.1029/JB073i020p06435

16. de la Fuente A, Shimizu K, Imberger J, Niño Y (2008) The evolution of internal waves in a rotating, stratified, circular basin and the influence of weakly nonlinear and nonhydrostatic accelerations. Limnol Oceanogr 53(6)(6):2738-2748. https://doi.org/10.4319/lo.2008.53.6.2738

17. de la Fuente A, Shimizu K, Niño Y, Imberger J (2010) Nonlinear and weakly nonhydrostatic inviscid evolution of internal gravitational basin-scale waves in a large, deep lake: lake constance. J Geophys Res Ocean. https://doi.org/10.1029/2009JC005839 
18. Fedorov A, Melville WK (1995) Propagation and breaking of nonlinear Kelvin wave. J Phys Oceanogr 25(11):2518-2531. https://doi.org/10.1175/1520-0485(1995)025\%3c2518:PABONK\%3e2.0.CO;2

19. Gill AE (1982) Atmosphere ocean dynamics. Academic Press, London

20. Grimshaw R, Helfrich K (2012) The effect of rotation on internal solitary waves. IMA J Appl Math 77(3):326-339. https://doi.org/10.1093/imamat/hxs024

21. Grimshaw RHJ (1985) Evolution equation for weakly nonlinear, long internal waves in a rotating fluid. Stud Appl Math 73(1):1-33. https://doi.org/10.1002/sapm19857311

22. Grimshaw RHJ, He JM, Ostrovsky LA (1998) Terminal damping of a solitary wave due to radiation in rotational systems. Stud Appl Math 101(2):197-210. https://doi.org/10.1111/1467-9590.00090

23. Grimshaw RHJ, Helfrich K (2008) Long-time solutions of the ostrovsky equation. Stud Appl Math 121(1):71-88. https://doi.org/10.1111/j.1467-9590.2008.00412.x

24. Grimshaw RHJ, Helfrich KR, Johnson ER (2013) Experimental study of the effect of rotation on nonlinear internal waves. Phys Fluids 25(5):056,602. https://doi.org/10.1063/1.4805092

25. Horn DA, Imberger J, Ivey GN (2001) The degeneration of large-scale interfacial gravity waves in lakes. J Fluid Mech 434:181-207. https://doi.org/10.1017/S0022112001003536

26. Huang KM, Zhang SD, Yi F, Huang CM, Gan Q, Gong Y, Zhang YH (2014) Nonlinear interaction of gravity waves in a nonisothermal and dissipative atmosphere. Ann Geophys 32(3):263-275. https://doi. org/10.5194/angeo-32-263-2014

27. Hutter K, Salvadè G, Spinedi C, Zamboni F, Bäuerle E (1991) Large scale water movements in lakes. Aquat Sci 53(2):100-135. https://doi.org/10.1007/BF00877057

28. Johnson ER, Grimshaw RHJ (2014) Geostrophic adjustment in a closed basin with islands. J Fluid Mech 738:358-377. https://doi.org/10.1017/jfm.2013.598

29. Joyce TM (1974) Nonlinear interactions among standing surface and internal gravity waves. J Fluid Mech 63(04):801. https://doi.org/10.1017/S0022112074002205

30. Lamb H (1932) Hydrodynamics, 6th edn. Dover, New York

31. Liu D, Ma Y, Dong G, Perlin M (2016) Detuning and wave breaking during nonlinear surface wave focusing. Ocean Eng 113:215-223. https://doi.org/10.1016/j.oceaneng.2015.12.048

32. MacIntyre S, Flynn KM, Jellison R, Romero JR (1999) Boundary mixing and nutrient fluxes in Mono lake, California. Limnol Oceanogr 44(3):512-529. https://doi.org/10.4319/lo.1999.44.3.0512

33. Maxworthy T (1983) Experiments in solitary internal Kelvin waves. J Fluid Mech. https://doi. org/10.1017/S0022112083000816

34. McGinnis DF, Lorke A, Wüest A, Stöckli A, Little JC (2004) Interaction between a bubble plume and the near field in a stratified lake. Water Resour Res. https://doi.org/10.1029/2004WR003038

35. Melville WK, Tomasson GG, Renouard DP (1989) On the stability of Kelvin waves. J Fluid Mech 206:1-23. https://doi.org/10.1017/S002211208900220X

36. Monismith SG (1985) Wind-forced motions in stratified lakes and their effect on mixed-layer shear. Limnol Oceanogr 30(4):771-783. https://doi.org/10.4319/lo.1985.30.4.0771

37. Mortimer CH (1974) Lake hydrodynamics. SIL Commun 1953-1996 20(1):124-197. https://doi. org/10.1080/05384680.1974.11923886

38. Münnich M, Wüest A, Imboden DM (1992) Observations of the second vertical mode of the internal seiche in an alpine lake. Limnol Oceanogr 37(8):1705-1719. https://doi.org/10.4319/lo.1992.37.8.1705

39. Ng SMY, Antenucci JP, Hipsey MR, Tibor G, Zohary T (2011) Physical controls on the spatial evolution of a dinoflagellate bloom in a large lake. Limnol Oceanogr 56(6):2265-2281. https://doi. org/10.4319/lo.2011.56.6.2265

40. Ostrovsky I, Yacobi YZ, Walline P, Kalikhman I (1996) Seiche-induced mixing: its impact on lake productivity. Limnol Oceanogr 41(2):323-332. https://doi.org/10.4319/lo.1996.41.2.0323

41. Pannard A, Beisner BE, Bird DF, Braun J, Planas D, Bormans M (2011) Recurrent internal waves in a small lake: potential ecological consequences for metalimnetic phytoplankton populations. Limnol Oceanogr Fluids Environ 1(1):91-109. https://doi.org/10.1215/21573698-1303296

42. Patterson JC, Hamblin PF, Imberger J (1984) Classification and dynamic simulation of the vertical density structure of lakes. Limnol Oceanogr 29(4):845-861. https://doi.org/10.4319/lo.1984.29.4.0845

43. Pedlosky J (1987) Geophysical fluid dynamics. Springer, New York

44. Preusse M, Peeters F, Lorke A (2010) Internal waves and the generation of turbulence in the thermocline of a large lake. Limnol Oceanogr 55(6):2353-2365. https://doi.org/10.4319/lo.2010.55.6.2353

45. Renouard DP, DHieres GC, Zhang X (1987) An experimental study of strongly nonlinear waves in a rotating system. J Fluid Mech 177:381-394. https://doi.org/10.1017/S0022112087001009

46. Rozas C, de la Fuente A, Ulloa H, Davies P, Niño Y (2014) Quantifying the effect of wind on internal wave resonance in Lake Villarrica, Chile. Environ Fluid Mech 14(4):849-871. https://doi.org/10.1007/ s10652-013-9329-9 
47. Sakai T, Redekopp LG (2010) A weakly nonlinear evolution model for long internal waves in a large lake. J Fluid Mech. https://doi.org/10.1017/S0022112010001114

48. Shimizu K, Imberger J (2009) Damping mechanisms of internal waves in constinuously stratified rotating basins. J Fluid Mech 637:137-172. https://doi.org/10.1017/S0022112009008039

49. Stocker R, Imberger J (2003) Energy partitioning and horizontal dispersion in a stratified rotating lake. J Phys Oceanogr 33:512-529. https://doi.org/10.1175/1520-0485(2003)033\%3C0512:EPAHD I\%3E2.0.CO;2

50. Tomasson GG (1988) On the stability of long nonlinear Kelvin waves. Master's thesis, Departmentof Civil Engineering, MIT

51. Tomasson GG, Melville WK (1990) Nonlinear and dispersive effects in Kelvin waves. Phys Fluids A 2(2):189-193. https://doi.org/10.1063/1.857768

52. Torrence C, Compo GP (1998) A practical guide to wavelet analysis. Bull Am Meteorol Soc 79(1):6178. https://doi.org/10.1175/1520-0477(1998)079\%3C0061:APGTWA\%3E2.0.CO;2

53. Ulloa HN, de la Fuente A, Niño Y (2014) An experimental study of the free evolution of rotating, nonlinear internal gravity waves in a two-layer stratified fluid. J Fluid Mech 742:308-339. https://doi. org/10.1017/jfm.2014.10

54. Ulloa HN, Winters KB, de la Fuente A, Niño Y (2015) Degeneration of internal Kelvin waves in a continuous two-layer stratification. J Fluid Mech 777:68-96. https://doi.org/10.1017/jfm.2015.311

55. Valipour R, Boegman L, Bouffard D, Rao YR (2017) Sediment resuspension mechanisms and their contributions to high-turbidity events in a large lake. Limnol Oceanogr 62(3):1045-1065. https://doi. org/10.1002/lno.10485

56. Vidal J, Casamitjana X, Colomer J, Serra T (2005) The internal wave field in sau reservoir: observation and modeling of a third vertical mode. Limnol Oceanogr 50(4):1326-1333. https://doi.org/10.4319/ 1o.2005.50.4.1326

57. Wake GW, Ivey GN, Imberger J (2005) The temporal evolution of baroclinic basin-scale waves in a rotating circular basin. J Fluid Mech 523:367-392. https://doi.org/10.1017/S0022112004002344

58. Wake GW, Ivey GN, Imberger J, McDonald NR, Stocker R (2004) Baroclinic geostrophic adjustment in a rotating circular basin. J Fluid Mech 515:63-86. https://doi.org/10.1017/S0022112004000230

59. Wüest A, Lorke A (2003) Small-scale hydrodynamics in lakes. Annu Rev Fluid Mech 35(1):373-412. https://doi.org/10.1146/annurev.fluid.35.101101.161220

\section{Affiliations}

\section{Pedro Rojas ${ }^{1} \cdot$ Hugo N. Ulloa ${ }^{2}$ D $\cdot$ Yarko Niño ${ }^{3}$}

Pedro Rojas

pedrojas@ing.uchile.cl

Yarko Niño

ynino@ing.uchile.cl

1 Departamento de Ingeniería Civil, Universidad de Chile, Santiago, Chile

2 Physics of Aquatic Systems Laboratory (APHYS) - Margaretha Kamprad Chair - École Polytechnique Fédérale de Lausanne, Lausanne, Switzerland

3 Departamento de Ingeniería Civil and Advanced Mining Technology Center, Universidad de Chile, Santiago, Chile 